\title{
LPV Control of a Tensegrity-Membrane System
}

\author{
Shu Yang ${ }^{1}$ \\ Northwestern Polytechnical University, Xi'an, Shaanxi 710072, China \\ Cornel Sultan ${ }^{2}$ \\ Virginia Polytechnic Institute and State University, Blacksburg, VA 24061, USA
}

\begin{abstract}
This article presents the details of designing a linear parameter-varying (LPV) controller for a tensegrity-membrane system. The major control objective is to deploy the system from its initial packaged configuration to its final deployed configuration. Since LPV synthesis naturally leads to an infinite-dimensional linear matrix inequality (LMI) problem, a gridding method is used to reduce it to a finite-dimensional LMI problem and the control design is performed using a control-oriented model based on the linearized system dynamics at a set of system equilibriums. To test the performance of the resulting controller, a nonlinear finite element model and the control-oriented model are used in numerical simulations. To test the robustness of the controller, sensor noise is considered in another nonlinear finite element simulation and the results are compared with the simulation results given by the nominal system.
\end{abstract}

Keywords: Tensegrity-membrane systems; Nonlinear finite element model; Control-oriented model; LPV control synthesis

\section{Introduction}

Gossamer spacecraft are a class of space systems which are lightweight and capable of changing system configurations significantly. Due to these two unique features, they can be packaged in small volume and transported by vehicles with limited payload and volume capacity. Membranes are key components of these space systems, usually serving as solar sail surfaces [1,2], antennas [3-5], reflectors [6], etc.

Traditional gossamer spacecraft are designed using rigid support frames composed primarily of bars to which tensioned membranes are attached. The mechanical behavior of these systems is mainly determined by the rigid support frames, and the membranes have little effect on the statics and dynamics of these structural systems. To change the configurations of such systems it is necessary to change the shape of the support frames. Therefore, numerous additional components, like actuators and rigid/flexible connecting parts, are required for the rigid support

\footnotetext{
${ }^{1}$ Assistant Professor, School of Automation, 127 West Youyi Road, Email: yangs530@yahoo.com; previously Graduate Research Assistant, Department of Aerospace and Ocean Engineering, Virginia Tech, Blacksburg, VA 24061.

${ }^{2}$ Associate Professor (Corresponding Author), Department of Aerospace and Ocean Engineering, 215 Randolph Hall, Email: csultan@vt.edu.
} 
frames to achieve system deployment. As a result, the weight of the overall system increases, diminishing the benefit of lightweightness due to the membranes. Moreover, the resulting systems turn out to be more complex, leading to higher risk of deployment failure [7]. To alleviate these problems, some gossamer spacecraft designs employ the inflation technology, leading to systems with membranes attached to inflatable support frames [8-11]. Such systems are free from mechanical connecting parts, like hinges and joints, but system configurations during inflation may not be accurately controlled. Therefore, additional control devices are required to achieve precise control of system components during deployment [12].

Tensegrity-membrane systems are a class of new bar-tendon-membrane structures. The key idea in creating such novel structures is to introduce membranes in tensegrity structures. The attached membranes in tensegritymembrane systems can be part of optical devices, antennas, etc., the bars provide sufficient system stiffness, and the tendons introduce tensile forces and some flexibility in the system to allow easy shape changes. Moreover, all of the system components can incorporate sensing mechanisms (e.g., via embedded optic fibers) and the tendons can also serve as actuators for active control. Since the actuators are embedded in the system, such a system is not affected by the drawbacks associated with additional mechanisms (e.g., latches, hinges, etc.) required for deployment which are typical for classical membrane systems with rigid support frames like the traditional gossamer structures mentioned before. On the other hand, bars in tensegrity-membrane systems improve their overall stiffness and enable more precise shape control compared to gossamer spacecraft based on inflatable structures. Therefore, tensegrity-membrane systems are intermediate solutions that may be able to alleviate key deficiencies of these two types of gossamer structures.

As mentioned previously, membranes play key roles in gossamer spacecraft and tensegrity-membrane systems. They can serve as reflectors in optical instruments, radar antennas, solar sails, etc. Membrane design, analysis, as well as its mechanical properties, have been studied by many researchers. Greschik and Mikulas [1] presented a design study of square solar sails and evaluated several classical sail design concepts. They also proposed their novel sail suspension design, named stripped sail architecture, and pointed out several design issues of sail structures deserving attention. Mikulas and Adler [2] provided a simplified approach for assessing square solar sails, which could be used in a preliminary design procedure. Kukathasan and Pellegrino [44] studied the vibration of tensioned membranes under air effects by performing finite element analysis, aiming to show the feasibility of using finite element analysis to simplify the testing of membrane structures. Adler et al. [45] studied the statics and dynamics of 
partially wrinkled membranes and presented several analysis results for different wrinkled membrane structures. Kukathasan and Pellegrino [46] conducted high-fidelity finite element simulations for wrinkled membranes. Thinshell elements were used for the membranes and a precise simulation of the static wrinkling process was performed. Wong and Pellegrino [47-49] studied wrinkled membranes based on experiments and finite element simulations. The analytical models used in their work were also presented and discussed. Recently, Reynolds and Murphey [50] presented a spiral folding concept for flat membrane apertures and tested its feasibility. Arya and Pellegrino [51] used a similar idea to study the deployment mechanics of highly compacted thin membrane structures.

Tensegrity systems are generally lightweight and capable of significant shape changes [13]. It has also been shown that the shapes of tensegrity systems can be accurately changed when appropriate controls are applied [1416]. As extensions of classical tensegrity systems, tensegrity-membrane systems inherit these major advantages, which enable these novel systems to be generally lightweight and to experience relatively easy folding and unfolding between packaged and erected/extended configurations. When controls are implemented, the configurations of tensegrity-membrane systems can be accurately controlled during deployment and no additional control devices or actuators are required to achieve this goal. This is a key improvement over inflatable gossamer spacecraft whose shape cannot be accurately controlled during inflation/deployment. Clearly, these mechanical properties make tensegrity-membrane systems promising candidates for lightweight, deployable space structures that can be used in space applications such as space antennas and solar sails, as well as in civil applications such as reconfigurable shelters, bridges, etc.

Controlled deployment for tensegrity systems has been studied by several researchers. A comprehensive survey of works in this area of can be found in [41]. More recent contributions include the deployment of a tensegrity-ring module [52] and the deployment of a tensegrity footbridge [53]. Also very recently Sultan [16] presented a deployment strategy based on infinitesimal mechanisms for tensegrity systems and accurate path tracking was achieved by implementing a robust nonlinear feedback controller. In the field of motion control for tensegrity robots, Kim et al. [54] adopted an open-loop control strategy to control the locomotion of tensegrity soft robots. The dynamic relaxation technique was also shown to be effective in the control of tensegrity soft robots for locomotion [55]. Hustig-Schultz et al. [56] used machine learning, feedback control via a neural network, central pattern generators, and genetic evolution to control the locomotion of tensegrity quadruped robots. 
As previously mentioned, appropriate controllers must be applied to tensegrity-membrane systems in order to achieve accurate system shape change. During deployment, a tensegrity-membrane system may move along a trajectory which covers a large region within the system's operational envelope. Therefore, during deployment a simple, linear controller may not provide good control performance. Similar issues are frequently encountered in many engineering problems (e.g., control design for most aerospace systems). The gain-scheduling control technique $[17,18]$ is commonly implemented in practice to schedule controllers designed at a set of system operational points using linearized system dynamics as functions of system parameters. It should be noted that engineering insight and ad hoc rules are required for controller scheduling. Moreover, since the gain-scheduling technique relies on discretized system operational regions, the resulting controllers do not guarantee the performance and stability of the closed-loop systems at the operational points other than the design points. These issues are the major motivation for the development of the linear parameter-varying (LPV) control technique.

LPV control synthesis has been studied by many researchers. For example, Tan et al. [19] studied quasi-LPV modeling of a generic missile and used the LPV synthesis technique to design an autopilot for the missile. Balas et al. [20] designed and analyzed LPV controllers for the dynamics of the F-14 aircraft in the lateral-directional axis during powered approach landing. Wu et al. [21] designed pitch-axis missile autopilots based on LPV control theory. This control approach not only captures nonlinear behaviors of plants, but also takes advantage of the welldeveloped analysis methods for linear systems, leading to optimal controllers with guaranteed robustness. Moreover, LPV synthesis provides controllers which are conceptually similar to the existing gain-scheduled controllers used in practice. In general, no significant complexity is introduced in terms of control implementation.

In [22], we designed a state-feedback LPV controller for a tensegrity-membrane system to achieve system deployment. The resulting controller displayed good performance in numerical simulations. However, the statefeedback LPV control theory requires that all states can be measured, which is not usually possible in practice. Moreover, measurement noise could not be directly included in the control synthesis presented in [22]. In this article, a major improvement over our previous work in [22] is the development of an output-feedback LPV controller for a tensegrity-membrane system to achieve system deployment. The impact of external perturbations and sensor noise on the closed-loop system is also considered in the design of this LPV controller, and frequencydependent weighting functions are used to achieve a trade-off between robustness and control performance. The LPV controller is designed using a control-oriented model of tensegrity-membrane systems presented in [23, 24]. 
This control-oriented model is developed based on several modeling assumptions in order to reduce model complexity. Clearly, a controller designed based on this simplified model should be tested using high-fidelity models.

Another major contribution of this article is that a nonlinear finite element model, which is reported in [25], is used to test the LPV controller designed in this work. This nonlinear finite element model is of great generality, since bars and membranes are considered as flexible bodies, and large deformations of system components can be captured by this model. The simulation results of the closed-loop system given by the nonlinear finite element model are also compared with the results given by the control-oriented model to investigate the reliability of the controloriented model. Moreover, sensor noise is considered in another nonlinear finite element simulation in order to test the robustness of the LPV controller.

\section{Tensegrity-Membrane System Description}

A tensegrity-membrane system is a prestressed system composed of bars, tendons, and membranes. In this article, we study a tensegrity-membrane system with $M$ stages, $N$ bars in each stage, and one membrane attached to the top of the system. This system is referred to as an $M-N$ tensegrity-membrane system. The bars are labeled $b_{i j}$, and a stage contains the bars with the same first index. For the $j$-th bar in the $i$-th stage, $b_{i j}$, the lower end and the upper end of bar $b_{i j}$ are labeled $A_{i j}$ and $B_{i j}$, respectively. The length of bar $b_{i j}$ is labeled $l_{i j}$. The system is located at a fixed base by connecting the lower ends of the bars in the first stage, i.e. $A_{1 j}$, to the base through frictionless rotational joints. The membrane is attached to the top of the system, and the membrane corners are connected to the top ends of the bars in the $M$-th stage. More details about the definition and the mechanical properties of tensegritymembrane systems can be found in [25] and [43].

To illustrate the shape of tensegrity-membrane systems, a system with two stages, three bars in each stage, and a membrane (a 2-3 tensegrity-membrane system) is depicted in Fig. 1. Note that the rotational joints located at bar ends $A_{1 j}$ are not depicted. 


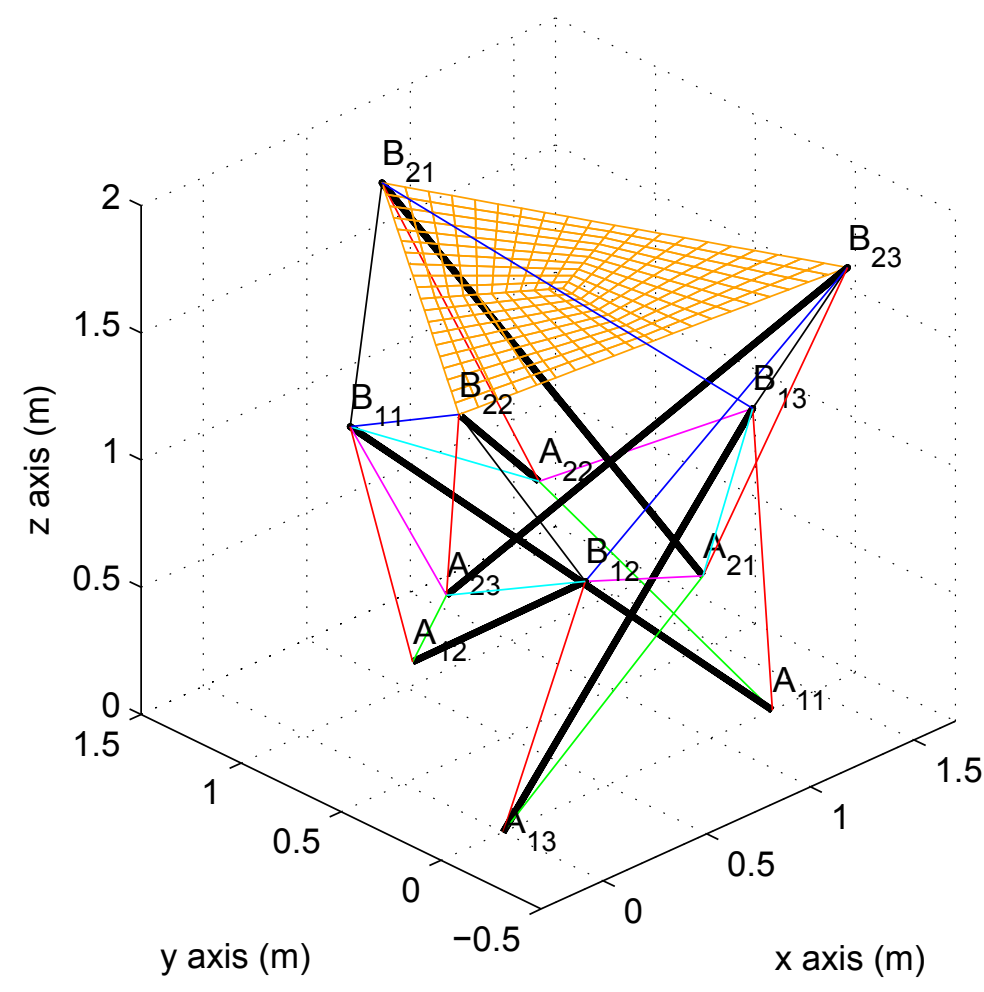

Fig. 1 The sketch of a two-stage three-bar tensegrity-membrane system

The inertial reference frame $O-x y z$ is fixed at the base of the system. The origin of $O-x y z$ is located at point $A_{1 N}$, i.e. $O \equiv A_{1 N}$, and the $O x$ axis goes through points $A_{1 N}$ and $A_{11}$. The $O y$ axis is perpendicular to the $O x$ axis in the base plane, and the $O z$ axis is perpendicular to the $O-x y$ plane, respecting the right-hand rule.

The tendon connection pattern used in this work is:

a) Tendon $S_{i j}$ connects the bar ends $A_{i j}$ and $B_{i, j-1}$

b) Tendon $V_{i j}$ connects the bar ends $A_{i j}$ and $B_{i-1, j+1}$

c) Tendon $D_{i j}$ connects the bar ends $A_{i j}$ and $B_{i-1, j-1}$

d) Tendon $C_{i j}$ connects the bar ends $A_{i j}$ and $A_{i-1, j-1}$

e) Tendon $P_{i j}$ connects the bar ends $B_{i j}$ and $B_{i-1, j}$

f) Tendon $R_{i j}$ connects the bar ends $B_{i j}$ and $B_{i-1, j-1}$

It should be pointed out that shape changes of tensegrity-membrane systems can be achieved using the tendon control technique, which alters tendon rest-lengths to change tendon forces. The tendon control technique has been 
used to control several structures such as tensegrity structures [14-16] and cellular trusses of aircraft morphing wings [57]. One way of implementing this technique in practice is to employ the motor-pulley-cable system. This approach is commonly used to control robot hands and arms [58-61] and can also be implemented to control tensegrity structures [62]. Another approach is to implement shape memory alloy (SMA) tendon wires, which have been shown to be effective in actuating biomimetic artificial fingers [63, 64] and soft morphing hands [65].

It should be noted that in many practical applications symmetric structures are desired, and it is also the case for most gossamer spacecraft designs. Therefore, symmetric tensegrity-membrane systems are of great importance and deserve the attention of engineers. A key requirement for symmetric tensegrity-membrane systems is that the declination angle $\theta_{i j}$ and azimuth angle $\psi_{i j}$ of each bar satisfy the following condition:

$$
\begin{aligned}
& \theta_{i j}=\theta_{i} ; \quad(i=1,2, \ldots, M ; j=1,2, \ldots, N) \\
& \psi_{i j}= \begin{cases}\psi_{i}+2 j \pi / N ; & (i=1,2, \ldots, M ; j=1,2, \ldots, N-1) \\
\psi_{i} ; & (i=1,2, \ldots, M ; j=N)\end{cases}
\end{aligned}
$$

where $\theta_{i}$ and $\psi_{i}$ are notations for the values of $\theta_{i j}$ and $\psi_{i j}$ for a bar $b_{i N}$ when a tensegrity-membrane system is at a symmetric equilibrium configuration. The bars in the same stage have the same length, i.e. $l_{i j}=l_{i}$. As a result, the bases of all stages are parallel to each other for a symmetric system. More details about symmetric tensegritymembrane systems and how such configurations can be computed in general can be found in [25].

In this work, we design symmetric system configurations with the property that $\theta_{i}$ is the same for all stages and so is $\psi_{i}$, which indicates that, for all $i=1,2, \ldots M$, (1) can be further expressed as:

$$
\begin{aligned}
\theta_{i j} & =\theta ; \quad(j=1,2, \ldots, N) \\
\psi_{i j} & = \begin{cases}\psi+2 j \pi / N ; & (j=1,2, \ldots, N-1) \\
\psi ; & (j=N)\end{cases}
\end{aligned}
$$

Also, the lengths of all bars are designed to be the same, i.e. $l_{i j}=l$. This symmetric equilibrium design requires fewer parameters to determine system symmetric configurations and reduces the cost of the related numerical computations.

\section{Mathematical Models}

As assemblies of membranes, bars, and tendons, tensegrity-membrane systems are flexible multibody systems. If these systems experience significant shape changes and large membrane deformations, they should be treated as nonlinear flexible multibody systems and studied using the nonlinear finite element method. However, if only the 
system dynamics around equilibriums is of interest, tensegrity-membrane systems can be treated as linear flexible multibody systems. Furthermore, low complexity, control-oriented models can be developed by using floating reference frames and the modal expansion technique. The resulting models have fewer generalized coordinates and may capture accurately the essence of system dynamics, so they may be suitable for control system design.

In this article, two mathematical models are used to conduct LPV control design and to test the resulting controller: a nonlinear finite element model and a control-oriented model. For the nonlinear finite element model, membrane elements are used to model the attached membrane, truss elements are used to model the bars, and the tendon effects are modeled as forces exerted on bar ends. For the control-oriented model, the bars are treated as rigid bodies, the geometrical nonlinearity of the attached membrane is ignored, and the linear finite element method is used to determine the terms related to the membrane elasticity.

\subsection{Nonlinear Finite Element Model}

To develop this nonlinear finite element model, the following modeling assumptions are made:

a) Linear elastic constitutive laws are used for the attached membranes, the bars, and the tendons.

b) The gravitational field is ignored. No external force is applied to the system.

c) A homogeneous membrane in the shape of a convex polygon in the undeformed configuration is used.

d) Since the membrane is very thin, its transverse bending stiffness and transverse shear stiffness are ignored.

e) The mechanics of wrinkles generated on the membrane is not modeled.

f) The rotational motion of each bar along its longitudinal axis is ignored.

g) The effect of tendons on the system is modeled as tendon forces exerted on bar ends. Tendon dynamics is ignored.

h) Tendons and the membrane cannot be compressed (i.e. they can carry only tensile loads).

The nonlinear finite element analysis used in this work follows the procedures introduced in [26]. The total Lagrangian formulation is applied and geometrical nonlinearity is considered. The strain and stress components are expressed in the curvilinear coordinate system $O-r_{1} r_{2} r_{3}$, and a tilde $(\sim)$ is placed over the terms expressed in the $O$ $r_{1} r_{2} r_{3}$ frame. Note that in this section tensor notations are used for simplicity. All of the coordinates and displacements are measured with respect to the inertial reference frame $O-x y z$ introduced in Section 2.

The principle of virtual work applied to the configuration at time $t+\Delta t$ is: 


$$
\int_{{ }_{0} V}{ }^{t+\Delta t} \tilde{S}^{i j} \delta^{t+\Delta t} \tilde{\varepsilon}_{i j} d{ }^{0} V={ }^{t+\Delta t} \mathscr{R}
$$

where ${ }_{0}^{t+\Delta t} \tilde{S}^{i j}$ is the contravariant component of the second Piola-Kirchhoff stress tensor at time $t+\Delta t$ with respect to the configuration at time 0 , and ${ }_{0}^{t+\Delta t} \tilde{\varepsilon}_{i j}$ is the covariant component of the Green-Lagrange strain at time $t+\Delta t$ with respect to time 0 . The term ${ }^{t+\Delta t} \mathcal{R}$ is the virtual work due to external forces and torques. The term ${ }^{0} V$ is the volume of the body at time 0 .

The stress and strain components can be decomposed as:

$$
{ }_{0}^{t+\Delta t} \tilde{S}^{i j}={ }_{0}^{t} \tilde{S}^{i j}+{ }_{0} \tilde{S}^{i j} ; \quad{ }_{0}^{t+\Delta t} \tilde{\varepsilon}_{i j}={ }_{0}^{t} \tilde{\varepsilon}_{i j}+{ }_{0} \tilde{\varepsilon}_{i j}
$$

In addition, the strain increment ${ }_{0} \tilde{\varepsilon}_{i j}$ can be written as the combination of a linear part ${ }_{0} \tilde{e}_{i j}$ and a nonlinear part ${ }_{0} \tilde{\eta}_{i j}$ :

$$
{ }_{0} \tilde{\varepsilon}_{i j}={ }_{0} \tilde{e}_{i j}+{ }_{0} \tilde{\eta}_{i j}
$$

After linearization, Eq. (3) becomes:

$$
\int_{{ }_{0}}{ }_{0} \tilde{C}^{i j r l}{ }_{0} \tilde{e}_{r l} \delta_{0} \tilde{e}_{i j} d^{0} V+\int_{{ }^{0} V}{ }_{0}^{t} \tilde{S}^{i j} \delta_{0} \tilde{\eta}_{i j} d^{0} V={ }^{t+\Delta t} \mathscr{R}-\int_{{ }^{0} V}{ }_{0}^{t} \tilde{S}^{i j} \delta_{0} \tilde{e}_{i j} d^{0} V
$$

where ${ }_{0} \tilde{C}^{i j r l}$ is the fourth-order contravariant constitutive tensor in $O-r_{1} r_{2} r_{3}$. Note that the virtual work ${ }^{t+\Delta t} \mathcal{R}$ includes the virtual work of inertia forces and damping forces. From Eq. (6), the incremental solution at $t+\Delta t$ can be computed. To improve the solution accuracy, an iterative procedure is applied, and the matrix equations for finite element analysis can be written as:

$$
\left\{\begin{array}{l}
\boldsymbol{M}^{t+\Delta t} \ddot{\boldsymbol{U}}^{(k)}+\left({ }_{0}^{t} \boldsymbol{K}_{\boldsymbol{L}}{ }^{(k-1)}+{ }_{0}^{t} \boldsymbol{K}_{N \boldsymbol{L}}{ }^{(k-1)}\right) \Delta \boldsymbol{U}^{(k)}={ }^{t+\Delta t} \boldsymbol{R}-{ }^{t+\Delta t} \boldsymbol{F}^{(k-1)} \\
{ }^{t+\Delta t} \boldsymbol{U}^{(k)}={ }^{t+\Delta t} \boldsymbol{U}^{(k-1)}+\Delta \boldsymbol{U}^{(k)} ;{ }^{t+\Delta t} \boldsymbol{U}^{(0)}={ }^{t} \boldsymbol{U}
\end{array}\right.
$$

where $\boldsymbol{M}$ is the mass matrix. Also $\boldsymbol{K}_{\boldsymbol{L}}$ and $\boldsymbol{K}_{N L}$ represent the linear strain incremental stiffness matrix and the nonlinear strain incremental stiffness matrix, respectively, $\boldsymbol{R}$ represents the vector of external forces and torques, $\boldsymbol{F}$ represents the vector of nodal point forces corresponding to the element stresses, and $\boldsymbol{U}$ represents the vector of nodal displacements.

For the nonlinear finite element model, the membrane element and the truss element presented in [26] are used to model the attached membrane and bars, respectively. We emphasize that in this work the tendons are always assumed to be in tension. According to modeling assumption h), tendons can only carry tensile forces. However bars can carry compressive loads and tensile loads. If the assumption that the tendons are always in tension is relaxed and 
the tendons are allowed to go slack, modeling them as bars in the nonlinear finite element model would not be possible, because the behavior of a slack tendon cannot be described by a bar element. As stated previously, the effect of tendons is modeled as forces exerted on bar ends, indicating that the dynamics of tendons, i.e. the dynamics of the actuators, is ignored. This simplification is commonly pursued in most research on control design for mechanical systems in the early stages and can significantly reduce the computational cost for the control problem studied in this work.

It should be pointed out that, since in this work the tendons are always assumed to be in tension, they can be modeled using multiple truss elements in order to capture their dynamics. Of course, to ensure correctness of the model, the condition that the tendons remain in tension during motion must be verified. In [43], we adopted this idea and developed a more complex nonlinear finite element model to study the mechanical properties of tensegritymembrane systems, such as system natural frequencies, system mode shapes, and the coupled dynamics of system components.

The coordinates of an arbitrary point in the four-node membrane element are:

$$
{ }^{t} x_{i}=\sum_{k=1}^{4} h_{k}{ }^{t} x_{i}^{k}
$$

where $h_{k}$ is the interpolation function for a four-node element:

$$
\begin{aligned}
& h_{k}=\frac{1}{4}\left(1+r_{1}^{k} r_{1}\right)\left(1+r_{2}^{k} r_{2}\right) ; \quad(k=1,2,3,4) \\
& \begin{cases}r_{1}^{1}=1 ; \quad r_{1}^{2}=-1 ; \quad r_{1}^{3}=-1 ; \quad r_{1}^{4}=1 \\
r_{2}=1 ; \quad r_{2}^{2}=1 ; \quad r_{2}^{3}=-1 ; \quad r_{2}^{4}=-1\end{cases} \\
& \left\{r_{2}^{1}=1 ; \quad r_{2}^{2}=1 ; \quad r_{2}^{3}=-1 ; \quad r_{2}^{4}=-1\right.
\end{aligned}
$$

The two-node truss element is used to model the tendons. The coordinates of a generic point in the two-node truss element are:

$$
{ }^{t} x_{i}=\sum_{k=1}^{2} h_{k}{ }^{t} x_{i}^{k}
$$

where $h_{k}$ is the interpolation function for a two-node truss element:

$$
h_{1}=\frac{1}{2}\left(1-r_{1}\right) ; \quad h_{2}=\frac{1}{2}\left(1+r_{1}\right)
$$




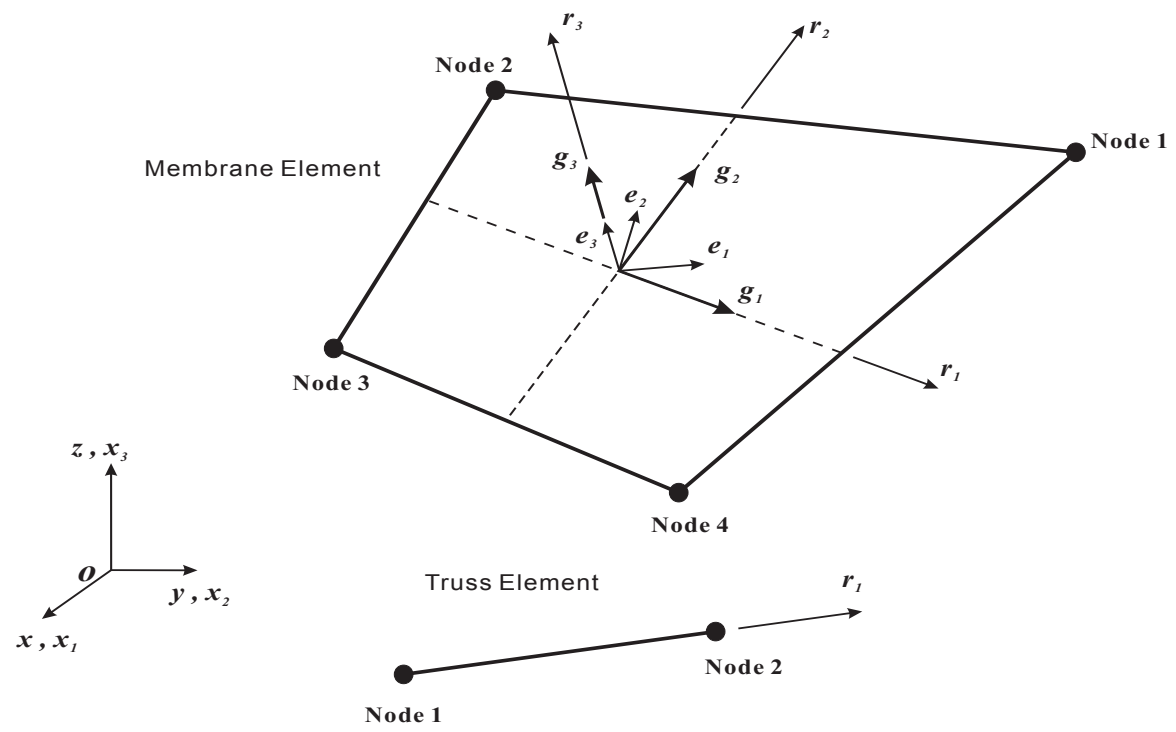

Fig. 2 The membrane element and the truss element

Using (8) and (10) at times $0, t$, and $t+\Delta t$, we can express the displacements as:

$$
\begin{aligned}
& { }^{t} u_{i}={ }^{t} x_{i}-{ }^{0} x_{i} \\
& u_{i}={ }^{t+\Delta t} x_{i}-{ }^{t} x_{i}
\end{aligned}
$$

After inserting (8) and (10) into (12), we obtain the displacements of the membrane element and the truss element:

$$
{ }^{t} u_{i}=\sum_{k=1}^{N D} h_{k}{ }^{t} u_{i}^{k} ; \quad u_{i}=\sum_{k=1}^{N D} h_{k} u_{i}^{k}
$$

where $N D$ is the number of nodes of an element, i.e. $N D=4$ for the membrane element, and $N D=2$ for the truss element. More details about this nonlinear finite element model can be found in [25].

\subsection{Control-Oriented Model}

For the control-oriented model, in addition to the modeling assumptions used for the nonlinear finite element model in Section 3.1, the following two modeling assumptions are made:

i) Bars are treated as rigid bodies.

j) The infinitesimal strain assumption is used for the attached membrane. In other words, the geometrical nonlinearity of the membrane is ignored.

These two assumptions reduce the number of generalized coordinates and the complexity of the control-oriented model, since the coordinates needed to model bar deformations are eliminated and the superposition principle, as 
well as the modal expansion technique, can be applied to the membrane. Note that the modeling assumption i) is commonly used in most research on tensegrity structures [13-16, 41].

For the bars in the first stage, since the lower end of bar $b_{1 j}$ is connected to the base, only two generalized coordinates, i.e. the declination angle, $\theta_{1 j}$, and the azimuth angle, $\psi_{1 j}$, are needed to determine the bar's orientation. For each bar $b_{i j}$ in the $i$-th stage $(i \geq 2)$, the location of its lower end, i.e. $x_{i j}, y_{i j}, z_{i j}$, and its declination and azimuth angle, $\theta_{i j}$ and $\psi_{i j}$, respectively, are used to determine the bar's location and orientation. Thus, the vector of generalized coordinates for bars is defined as:

$$
\boldsymbol{q}=\left[\begin{array}{llllllll}
\theta_{11} & \psi_{11} & \cdots & x_{M N} & y_{M N} & z_{M N} & \theta_{M N} & \psi_{M N}
\end{array}\right]^{T}
$$

Note that $\theta_{i j} \in(0, \pi / 2)$ and $\psi_{i j} \in(-\pi, \pi)$. The generalized coordinates for bar $b_{i j}$ are depicted in Fig. 3 .

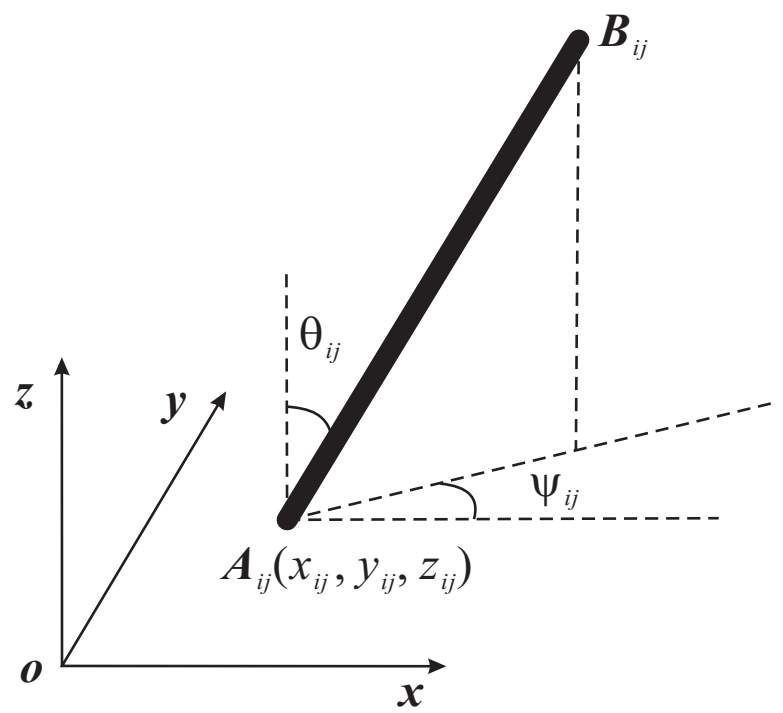

Fig. 3 Generalized coordinates of bar $b_{i j}$

In order to model the attached membrane, we first introduce a floating reference frame labeled $O_{f}-x_{f} y_{f} z_{f}$. The origin of $O_{f}-x_{f} y_{f} z_{f}$ is located at the point $B_{M N}$, i.e. $O_{f} \equiv B_{M N}$. The plane $O_{f} x_{f} y_{f}$ is identical with the plane determined by $B_{M N}, B_{M 1}$, and $B_{M, N-I}$. The axis $O_{f} x_{f}$ goes through $B_{M N}$ and $B_{M 1}$. The axis $O_{f} y_{f}$ is perpendicular to the axis $O_{f} x_{f}$, and the $O_{f} z_{f}$ axis can be determined based on the right-hand rule. According to the definition of reference frames, $O_{f}-x_{f} y z_{f}$ is fixed on the undeformed membrane.

For a generic point $P_{i}$ on the membrane, three variables $u, v$, and $w$ are used to describe its deflections in the $x_{f}$, $y_{f}$, and $z_{f}$ axes. Based on the method used in [27], the deflections of the membrane, $u, v$, and $w$, are separated into 
two parts: the prestressing deflections, $u^{s}, v^{s}$, and $w^{s}$, and the relative deflections, $u^{r}, v^{r}$, and $w^{r}$. Modal coordinates are used to discretize the relative deflections. Thus, we can express the deflections in the following form:

$$
\begin{aligned}
{\left[\begin{array}{lll}
u & v & w
\end{array}\right]^{T} } & =\left[\begin{array}{lll}
u^{s}+u^{r} & v^{s}+v^{r} & w^{s}+w^{r}
\end{array}\right]^{T} \\
& =\left[\begin{array}{lll}
u^{s}+\sum_{i=1}^{K} \phi_{u}^{i} \eta_{i} & v^{s}+\sum_{i=1}^{K} \phi_{v}^{i} \eta_{i} & w^{s}+\sum_{i=1}^{K} \phi_{w}^{i} \eta_{i}
\end{array}\right]^{T}
\end{aligned}
$$

where $\eta_{i}$ is the modal coordinate and $\phi_{u}^{i}, \phi_{v}^{i}, \phi_{w}^{i}$ are mode shapes of the relative deflections.

The variables $u$ and $v$ in (15) are commonly known as the in-plane deflections and $w$ is known as the transverse deflection (i.e. the out-of-plane deflection). As mentioned previously, these three variables are used to model the vibrations of the attached membrane in three dimensions. Note that the transverse vibration modes of membranes are usually of lower frequencies and more easily to be excited than the in-plane vibration modes, indicating that the transverse vibration of the attached membrane could be more easily coupled with the dynamics of other system components of a tensegrity-membrane system. Therefore, the membrane transverse vibration needs to be accurately modeled in order to study its influence to the dynamics of the tensegrity-membrane system, whereas the in-plane vibration modes could be neglected according to the modal truncation technique. For more details about the modal analysis of tensegrity-membrane systems and the attached membranes, readers are referred to [43] and [23].

The independent generalized coordinates can be expressed as:

$$
\hat{z}=\left[\begin{array}{llll}
\boldsymbol{q}^{T} & \eta_{1} & \cdots & \eta_{K}
\end{array}\right]^{T}
$$

Note that the relative deflections of a generic point on the membrane, $P_{i}\left(x_{f}^{i}, y_{f}^{i}\right)$, can be expressed as (see (15)):

$$
u_{i}^{r}=\sum_{j=1}^{K} \phi_{u}^{j}\left(x_{f}^{i}, y_{f}^{i}\right) \eta_{j} ; \quad v_{i}^{r}=\sum_{j=1}^{K} \phi_{v}^{j}\left(x_{f}^{i}, y_{f}^{i}\right) \eta_{j} ; \quad w_{i}^{r}=\sum_{j=1}^{K} \phi_{w}^{j}\left(x_{f}^{i}, y_{f}^{i}\right) \eta_{j}
$$

According to (17), we can find $K$ points on the membrane and get $3 K$ membrane relative deflections. Then, if the first $K$ modes are used, we can choose $K$ components from the $3 K$ membrane relative deflections and rearrange (17) as:

$$
\begin{aligned}
\boldsymbol{v} & =\boldsymbol{T}_{\boldsymbol{v} \boldsymbol{\eta}} \boldsymbol{\eta} \\
\boldsymbol{v} & =\left[\begin{array}{lllllllll}
u_{1}^{r} & \cdots & u_{k_{1}}^{r} & v_{1}^{r} & \cdots & v_{k_{2}}^{r} & w_{1}^{r} & \cdots & w_{k_{3}}^{r}
\end{array}\right]^{T} ; \quad\left(k_{1}+k_{2}+k_{3}=K\right) \\
\boldsymbol{\eta} & =\left[\begin{array}{lll}
\eta_{1} & \cdots & \eta_{K}
\end{array}\right]^{T}
\end{aligned}
$$


These $K$ points and the corresponding membrane relative deflections can be chosen to ensure that matrix $\boldsymbol{T}_{v \boldsymbol{\eta}}$ is invertible. Thus, these $K$ membrane relative deflections can be used as generalized coordinates instead of the modal coordinates, i.e. another set of generalized coordinates is:

$$
\boldsymbol{z}=\left[\begin{array}{ll}
\boldsymbol{q}^{T} & \boldsymbol{v}^{T}
\end{array}\right]^{T}
$$

The equations of motion for tensegrity-membrane systems can be developed using Lagrange's equations. Holonomic constraints and Lagrange's multipliers are used to facilitate the mathematical derivation [28]. The resulting equations of motion are a set of differential-algebraic equations (DAEs). After standard mathematical manipulations involving coordinate partitioning and coordinate mapping (see [24] for details), these DAEs can be converted into ordinary differential equations (ODEs), which can be written as:

$$
\ddot{z}+\boldsymbol{F}_{z}(z, \dot{z})+\boldsymbol{B}_{z}(z) \boldsymbol{u}=\boldsymbol{O}
$$

where $\boldsymbol{u}$ is the vector of tendon rest-lengths and the independent generalized coordinates in (19) are used. More details about this control-oriented model can be found in [23, 24].

\section{LPV Control Synthesis}

As mentioned previously, the development of the LPV control technique is motivated by the issues associated with the classical gain-scheduling control technique. A major advantage of LPV synthesis is that the resulting robust controllers can guarantee the control performance within regions between control design points. Moreover, the LPV control technique, which conceptually fits into the gain-scheduling framework, does not introduce additional complexity to the resulting controllers compared to the existing gain-scheduled controllers used in practice. Therefore, LPV control synthesis provides an efficient approach to the control design for nonlinear physical systems.

In this section, we provide a brief overview of the key concepts of LPV synthesis. Let the symbol $\mathbb{R}$ represent the set of real numbers. For a compact set $\mathcal{P} \subset \mathbb{R}^{s}$, the parameter variation set $\mathcal{F}_{\mathcal{P}}$ denotes the set of all continuously differentiable functions mapping $\mathbb{R}$ (time) into $\mathcal{P}$. A generic LPV system (e.g., the open-loop plant model) is expressed as:

$$
\begin{aligned}
& {\left[\begin{array}{c}
\dot{\boldsymbol{x}}(t) \\
\boldsymbol{e}(t) \\
\boldsymbol{y}(t)
\end{array}\right]=\left[\begin{array}{lll}
A(\rho(t)) & B_{1}(\rho(t)) & B_{2}(\rho(t)) \\
C_{1}(\rho(t)) & D_{11}(\rho(t)) & D_{12}(\rho(t)) \\
C_{2}(\rho(t)) & D_{21}(\rho(t)) & D_{22}(\rho(t))
\end{array}\right]\left[\begin{array}{l}
\boldsymbol{x}(t) \\
d(t) \\
\boldsymbol{u}(t)
\end{array}\right]} \\
& \rho \in \mathcal{F}_{\mathcal{P}}, \quad \rho(t) \in \mathcal{P}
\end{aligned}
$$


where $\boldsymbol{x}(t)$ is the vector of state variables, $\boldsymbol{d}(t)$ is the vector of generalized inputs, $\boldsymbol{u}(t)$ is the vector of control inputs, $\boldsymbol{e}(t)$ is the vector of generalized errors, and $\boldsymbol{y}(t)$ is the vector of measured outputs. Note that an LPV system like (21) can be developed from a nonlinear model of a physical system (e.g., a tensegrity-membrane system) via different approaches, such as linearization, state transformation, function substitution, etc. [42]. Without loss of generality, it is assumed that $\boldsymbol{D}_{22}(\rho(t))=\boldsymbol{O}$. This assumption can be satisfied by changing controller parameters and greatly simplifies mathematical derivations [34].

A generic LPV controller is expressed as:

$$
\begin{aligned}
& \dot{\boldsymbol{x}}_{\boldsymbol{k}}=\boldsymbol{A}_{\boldsymbol{k}}(\rho(t)) \boldsymbol{x}_{\boldsymbol{k}}+\boldsymbol{B}_{\boldsymbol{k}}(\rho(t)) \boldsymbol{y} \\
& \boldsymbol{u}=\boldsymbol{C}_{\boldsymbol{k}}(\rho(t)) \boldsymbol{x}_{\boldsymbol{k}}+\boldsymbol{D}_{\boldsymbol{k}}(\rho(t)) \boldsymbol{y}
\end{aligned}
$$

By combining (21) and (22) the closed-loop LPV system can be written as:

$$
\left[\begin{array}{c}
\dot{\boldsymbol{x}}(t) \\
\boldsymbol{e}(t)
\end{array}\right]=\left[\begin{array}{ll}
\boldsymbol{A}_{c l}(\rho(t)) & \boldsymbol{B}_{c l}(\rho(t)) \\
\boldsymbol{C}_{c l}(\rho(t)) & \boldsymbol{D}_{c l}(\rho(t))
\end{array}\right]\left[\begin{array}{l}
\boldsymbol{x}(t) \\
\boldsymbol{d}(t)
\end{array}\right]
$$

where

$$
\begin{aligned}
& \boldsymbol{A}_{c l}(\rho(t))=\left[\begin{array}{cc}
\boldsymbol{A}(\rho(t))+\boldsymbol{B}_{2}(\rho(t)) \boldsymbol{D}_{\boldsymbol{k}}(\rho(t)) \boldsymbol{C}_{2}(\rho(t)) & \boldsymbol{B}_{2}(\rho(t)) \boldsymbol{C}_{\boldsymbol{k}}(\rho(t)) \\
\boldsymbol{B}_{k}(\rho(t)) \boldsymbol{C}_{2}(\rho(t)) & \boldsymbol{A}_{\boldsymbol{k}}(\rho(t))
\end{array}\right] \\
& \boldsymbol{B}_{c l}(\rho(t))=\left[\begin{array}{c}
\boldsymbol{B}_{1}(\rho(t))+\boldsymbol{B}_{2}(\rho(t)) \boldsymbol{D}_{k}(\rho(t)) \boldsymbol{D}_{21}(\rho(t)) \\
\boldsymbol{B}_{k}(\rho(t)) \boldsymbol{D}_{21}(\rho(t))
\end{array}\right] \\
& C_{c l}(\rho(t))=\left[C_{1}(\rho(t))+D_{12}(\rho(t)) D_{k}(\rho(t)) C_{2}(\rho(t)) \quad D_{12}(\rho(t)) C_{k}(\rho(t))\right] \\
& D_{c l}(\rho(t))=D_{11}(\rho(t))+D_{12}(\rho(t)) D_{k}(\rho(t)) D_{21}(\rho(t))
\end{aligned}
$$

The induced $L_{2}$-norm of the transfer function of the closed-loop system $\boldsymbol{G}_{\boldsymbol{e}}$ is defined as:

$$
\left\|\boldsymbol{G}_{\boldsymbol{e d}}\right\|=\sup _{\boldsymbol{\rho} \in \mathcal{F}_{\mathcal{P}}} \sup _{\|\boldsymbol{d}\|_{2} \neq 0, \boldsymbol{d} \in L_{2}} \frac{\|\boldsymbol{e}\|_{2}}{\|\boldsymbol{d}\|_{2}}
$$

Here $\left\|^{*}\right\|_{2}$ denotes the standard $L_{2}$ norm of signals. If $\boldsymbol{d}$ is an $N \times 1$ vector, $L_{2}$ in (25) is the Hilbert space of real $N$ dimensional signals of finite $L_{2}$ norm (i.e. finite energy) defined on the positive real axis.

A key result in LPV synthesis is the following theorem [29]:

Theorem 4.1. For all $\rho(t) \in \mathcal{P}$ and the open-loop LPV plant in (21), matrix $\boldsymbol{A}_{c l}(\rho(t))$ of the closed-loop system in (23) is exponentially stable and there is an upper bound $\gamma$ on $\left\|\boldsymbol{G}_{\boldsymbol{e d}}\right\|$, if there exists a symmetric positive-definite matrix $\boldsymbol{W}(\rho(t))$, such that: 


$$
\left[\begin{array}{ccc}
\boldsymbol{A}_{c l}{ }^{T}(\rho(t)) \boldsymbol{W}(\rho(t))+\boldsymbol{W}(\rho(t)) \boldsymbol{A}_{c l}(\rho(t))+\frac{d \boldsymbol{W}(\rho(t))}{d t} & \boldsymbol{W}(\boldsymbol{\rho}(t)) \boldsymbol{B}_{c l}(\rho(t)) & \boldsymbol{C}_{c l}{ }^{T}(\rho(t)) \\
\boldsymbol{B}_{c l}{ }^{T}(\rho(t)) \boldsymbol{W}(\rho(t)) & -\gamma \boldsymbol{I} & \boldsymbol{D}_{c l}{ }^{T}(\rho(t)) \\
\boldsymbol{C}_{c l}(\rho(t)) & \boldsymbol{D}_{c l}(\rho(t)) & -\gamma \boldsymbol{I}
\end{array}\right] \prec \boldsymbol{O}
$$

Theorem 4.1 is the generalization of the well-known Bounded Real Lemma for LPV systems. Pre-multiplying and post-multiplying (26) by $\operatorname{diag}\left(\boldsymbol{W}(\rho(t))^{-1}, \boldsymbol{I}, \boldsymbol{I}\right)$, and letting $\boldsymbol{X}(\boldsymbol{\rho}(t))=\boldsymbol{W}(\rho(t))^{-1}$, we can convert (26) into the following LMI:

$$
\left[\begin{array}{ccc}
\boldsymbol{X}(\rho(t)) \boldsymbol{A}_{c l}(\rho(t))^{T}+\boldsymbol{A}_{c l}(\rho(t)) \boldsymbol{X}(\rho(t))-\frac{d \boldsymbol{X}(\rho(t))}{d t} & \boldsymbol{B}_{c l}(\rho(t)) & \boldsymbol{X}(\rho(t)) \boldsymbol{C}_{c l}{ }^{T}(\rho(t)) \\
\boldsymbol{B}_{c l}{ }^{T}(\rho(t)) & -\gamma \boldsymbol{I} & \boldsymbol{D}_{c l}{ }^{T}(\rho(t)) \\
\boldsymbol{C}_{c l}(\rho(t)) \boldsymbol{X}(\rho(t)) & \boldsymbol{D}_{c l}(\rho(t)) & -\gamma \boldsymbol{I}
\end{array}\right] \prec \boldsymbol{O}
$$

Matrices $\boldsymbol{X}(\rho(t))$ and $\boldsymbol{X}^{1}(\rho(t))$ can be parametrized as:

$$
X(\rho(t))=\left[\begin{array}{cc}
R(\rho(t)) & M(\rho(t)) \\
M(\rho(t))^{T} & U(\rho(t))
\end{array}\right] ; \quad X^{-1}(\rho(t))=\left[\begin{array}{cc}
S(\rho(t)) & N(\rho(t)) \\
N(\rho(t))^{T} & V(\rho(t))
\end{array}\right]
$$

where $\boldsymbol{R}(\rho(t))$ and $\boldsymbol{S}(\rho(t))$ are symmetric positive-definite matrices. Based on the identity $\boldsymbol{X}(\rho(t)) \boldsymbol{X}^{1}(\rho(t))=\boldsymbol{I}$, it can be found that

$$
\boldsymbol{I}-\boldsymbol{R}(\rho(t)) \boldsymbol{S}(\rho(t))=\boldsymbol{M}(\rho(t)) \boldsymbol{N}(\rho(t))^{T}
$$

Thus, two matrices, namely $\Pi_{1}(\rho(t))$ and $\Pi_{2}(\rho(t))$, are defined as:

$$
\Pi_{1}(\rho(t))=\left[\begin{array}{cc}
\boldsymbol{R}(\rho(t)) & \boldsymbol{I} \\
\boldsymbol{M}(\rho(t))^{T} & \boldsymbol{O}
\end{array}\right] ; \quad \Pi_{2}(\rho(t))=\left[\begin{array}{cc}
\boldsymbol{I} & \boldsymbol{S}(\rho(t)) \\
\boldsymbol{O} & \boldsymbol{N}(\rho(t))^{T}
\end{array}\right]
$$

It can be easily verified that $X(\rho(t)) \Pi_{2}(\rho(t))=\Pi_{1}(\rho(t))$. After pre-multiplying and post-multiplying (27) by $\operatorname{diag}\left(\Pi_{2}(\rho(t)){ }^{T}, \boldsymbol{I}, \boldsymbol{I}\right)$ and $\operatorname{diag}\left(\Pi_{2}(\rho(t)), \boldsymbol{I}, \boldsymbol{I}\right)$, respectively, we can determine the LPV controller in (22) by solving the following convex optimization problem [30]:

$$
\begin{aligned}
\text { find } & \boldsymbol{R}(\rho(t)), \boldsymbol{S}(\rho(t)), \overline{\boldsymbol{A}}_{\boldsymbol{k}}(\rho(t)), \overline{\boldsymbol{B}}_{\boldsymbol{k}}(\rho(t)), \overline{\boldsymbol{C}}_{\boldsymbol{k}}(\rho(t)) \text {, and } \boldsymbol{D}_{\boldsymbol{k}}(\rho(t)) \\
\text { subject to } & {\left[\begin{array}{cc}
\boldsymbol{R}(\rho(t)) & \boldsymbol{I} \\
\boldsymbol{I} & \boldsymbol{S}(\rho(t))
\end{array}\right] \succ \boldsymbol{O} } \\
& {\left[\begin{array}{ccc}
\dot{\Psi}+\Psi_{\mathbf{A}}+\Psi_{\mathrm{A}}{ }^{T} & \Psi_{\mathbf{B}} & \Psi_{\mathrm{C}}{ }^{T} \\
\boldsymbol{\Psi}_{\mathbf{B}}{ }^{T} & -\gamma \boldsymbol{I} & \Psi_{\mathbf{D}}{ }^{T} \\
\Psi_{\mathrm{C}} & \Psi_{\mathbf{D}} & -\gamma \boldsymbol{I}
\end{array}\right] \prec \boldsymbol{O} }
\end{aligned}
$$

where 


$$
\begin{aligned}
& \Psi_{\mathrm{A}}=\left[\begin{array}{cc}
\boldsymbol{A}(\rho(t)) \boldsymbol{R}(\rho(t))+\boldsymbol{B}_{\mathbf{2}}(\rho(t)) \overline{\boldsymbol{C}}_{\boldsymbol{k}}(\rho(t)) & \boldsymbol{A}(\rho(t))+\boldsymbol{B}_{\mathbf{2}}(\rho(t)) \boldsymbol{D}_{\boldsymbol{k}}(\rho(t)) \boldsymbol{C}_{\mathbf{2}}(\rho(t)) \\
\overline{\boldsymbol{A}}_{\boldsymbol{k}}(\rho(t)) & \boldsymbol{S}(\rho(t)) \boldsymbol{A}(\rho(t))+\overline{\boldsymbol{B}}_{\boldsymbol{k}}(\rho(t)) \boldsymbol{C}_{\mathbf{2}}(\rho(t))
\end{array}\right] \\
& \Psi_{\mathrm{B}}=\left[\begin{array}{c}
\boldsymbol{B}_{1}(\rho(t))+\boldsymbol{B}_{2}(\rho(t)) \boldsymbol{D}_{k}(\rho(t)) \boldsymbol{D}_{21}(\rho(t)) \\
\boldsymbol{S}(\rho(t)) \boldsymbol{B}_{1}(\rho(t))+\overline{\boldsymbol{B}}_{\boldsymbol{k}}(\rho(t)) \boldsymbol{D}_{21}(\rho(t))
\end{array}\right] \\
& \Psi_{\mathrm{C}}=\left[\boldsymbol{C}_{1}(\rho(t)) \boldsymbol{R}(\rho(t))+D_{12}(\rho(t)) \overline{\boldsymbol{C}}_{\boldsymbol{k}}(\rho(t)) \quad C_{1}(\rho(t))+D_{12}(\rho(t)) \boldsymbol{D}_{k}(\rho(t)) \boldsymbol{C}_{2}(\rho(t))\right] \\
& \Psi_{\mathrm{D}}=\boldsymbol{D}_{11}(\rho(t))+D_{12}(\rho(t)) \boldsymbol{D}_{k}(\rho(t)) \boldsymbol{D}_{21}(\rho(t)) \\
& \dot{\Psi}=\left[\begin{array}{cc}
\dot{\boldsymbol{R}}(\rho(t)) & \boldsymbol{O} \\
\boldsymbol{O} & -\dot{\boldsymbol{S}}(\rho(t))
\end{array}\right]
\end{aligned}
$$

The controller in (22) can be found using the following equations:

$$
\begin{aligned}
& M N^{T}=I-R S \\
& B_{k}=N^{-1}\left(\bar{B}_{k}-S B_{2} D_{k}\right) \\
& C_{k}=\left(\bar{C}_{k}-D_{k} C_{2} R\right) M^{-T} \\
& A_{k}=N^{-1}\left(\bar{A}_{k}-N B_{k} C_{2} R-S B_{2} C_{k} M^{T}-S\left(A+B_{2} D_{k} C_{2}\right) R+R \dot{S}+M \dot{N}^{T}\right) M^{-T}
\end{aligned}
$$

Note that the argument of each matrix in (33) (i.e. $\rho(t))$ is not written for clarity of the expressions.

The LMI in (31) imposes an infinite number of constraints on matrices $\boldsymbol{R}(\rho(t)), \boldsymbol{S}(\rho(t)), \overline{\boldsymbol{A}}_{\boldsymbol{k}}(\rho(t)), \overline{\boldsymbol{B}}_{\boldsymbol{k}}(\rho(t))$, $\overline{\boldsymbol{C}}_{\boldsymbol{k}}(\rho(t))$, and $\boldsymbol{D}_{\boldsymbol{k}}(\rho(t))$, since these constraints must be satisfied for all trajectories $\boldsymbol{\rho}(t)$ in the set $\mathcal{P}$. In this work, this problem is relaxed by discretizing the set $\mathcal{P}$ via a gridding approach [29].

\section{Control Design}

In this work, we use the control-oriented model to conduct LPV control design for a 2-3 symmetric tensegritymembrane system. The first four modes are used to model membrane vibrations, and modal analysis results show that these four modes are all transverse vibration modes. Therefore, based on the system definitions in Section 2 and the generalized coordinates defined in Section 3.2, vector $\boldsymbol{q}$ in (19) is a $21 \times 1$ vector, vector $\boldsymbol{v}$ in (19) is a $4 \times 1$ vector, and vector $\boldsymbol{u}$ in (20) is a $21 \times 1$ vector.

The design objectives for the LPV controller are characterized as follows:

1. Deployment requirement: the locations and attitudes of bars, $\boldsymbol{q}$, track the desired trajectory, $\boldsymbol{q}_{\boldsymbol{d}}$ In this work, $\boldsymbol{q}_{\boldsymbol{d}}$ is treated as a time-varying signal. Note that, for some symbols representing functions, we do not write the corresponding independent variables for clarity. For example, we use $\boldsymbol{q}_{\boldsymbol{d}}$ instead of $\boldsymbol{q}_{\boldsymbol{d}}(t)$ in this article. 
2. Membrane vibration requirement: the dynamics of the attached membrane can be stabilized at equilibrium, i.e. $\boldsymbol{v}_{\boldsymbol{d}}=\boldsymbol{O}$.

3. Tendon requirement: the tendon rest-lengths do not exceed the corresponding tendon lengths. In other words, tendon lengths are treated as control limits and tendons do not go slack.

The definition of symmetric tensegrity-membrane systems in (2) indicates that $\theta$ and $\psi$ can specify symmetric equilibrium configurations. Based on this definition, it can be easily found that $\theta$ and $\psi$ correspond to the declination angle and azimuth angle of bar $b_{13}$, i.e. $\theta=\theta_{13}$ and $\psi=\psi_{13}$. Therefore, two scheduling parameters are chosen as: $\rho_{1}=$ $\theta_{13}$ and $\rho_{2}=\psi_{13}$. Since $\rho_{1}$ and $\rho_{2}$ are state variables, the 2-3 tensegrity-membrane system is represented by a quasiLPV model. It is known that the methods used to design LPV controllers can be applied to quasi-LPV models [31].

For this example, the parameter space $\mathcal{P}$ is defined as: $\mathcal{P}=\rho_{1} \times \rho_{2}=\left[63^{\circ}, 74.46^{\circ}\right] \times\left[7.5^{\circ}, 9.22^{\circ}\right]$. The parameter space is discretized by a $5 \times 3$ grid, which corresponds to 15 system symmetric equilibriums. The discretized parameter space is: $\mathcal{P}_{\mathcal{D}}=\left\{63^{\circ}, 65.86^{\circ}, 68.73^{\circ}, 71.59^{\circ}, 74.46^{\circ}\right\} \times\left\{7.5^{\circ}, 8.36^{\circ}, 9.22^{\circ}\right\}$, which is a discretization of $\mathcal{P}$. The envelope of the parameter space (i.e. the envelope of $\mathcal{P}$ ) and the control design grid (i.e. $\mathcal{P}_{\mathcal{D}}$ ) are plotted in Fig. 4. Note that a much finer grid, named the verification grid, is also plotted in Fig. 4. This grid is used for LPV controller verification. More details about this grid can be found in the following section. 


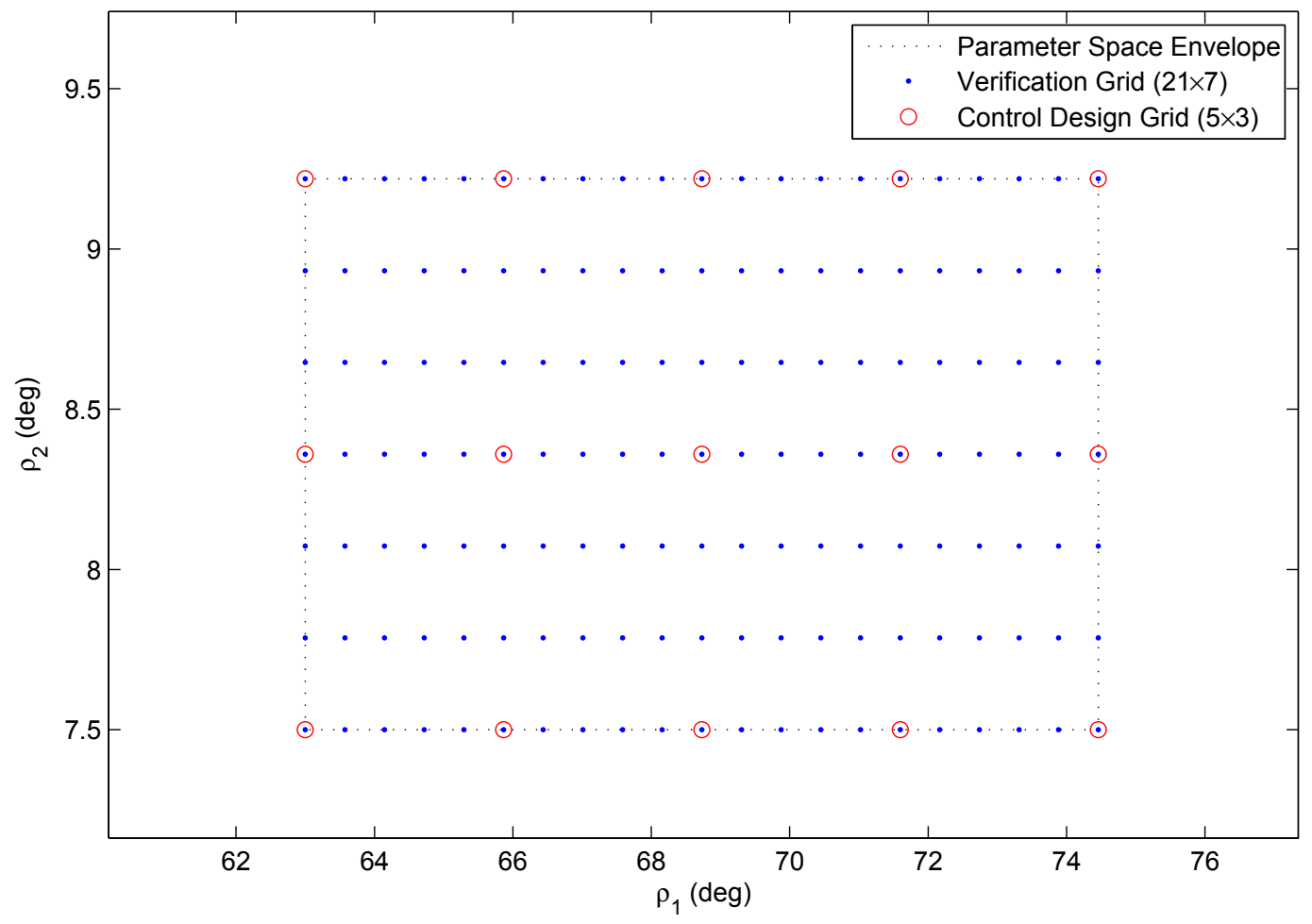

Fig. 4 Parameter space and discretized grids

After linearizing (20) at these equilibriums, we get 15 linearized first order models, whose state variables can be expressed as: $\boldsymbol{x}=\left[\begin{array}{cc}z^{T} & \dot{\boldsymbol{z}}^{T}\end{array}\right]^{T}$. Note that we use the same symbols to represent the variables in the nonlinear equations of motion and the corresponding perturbed variables in the linearized models. There should be no confusion with this notation. 


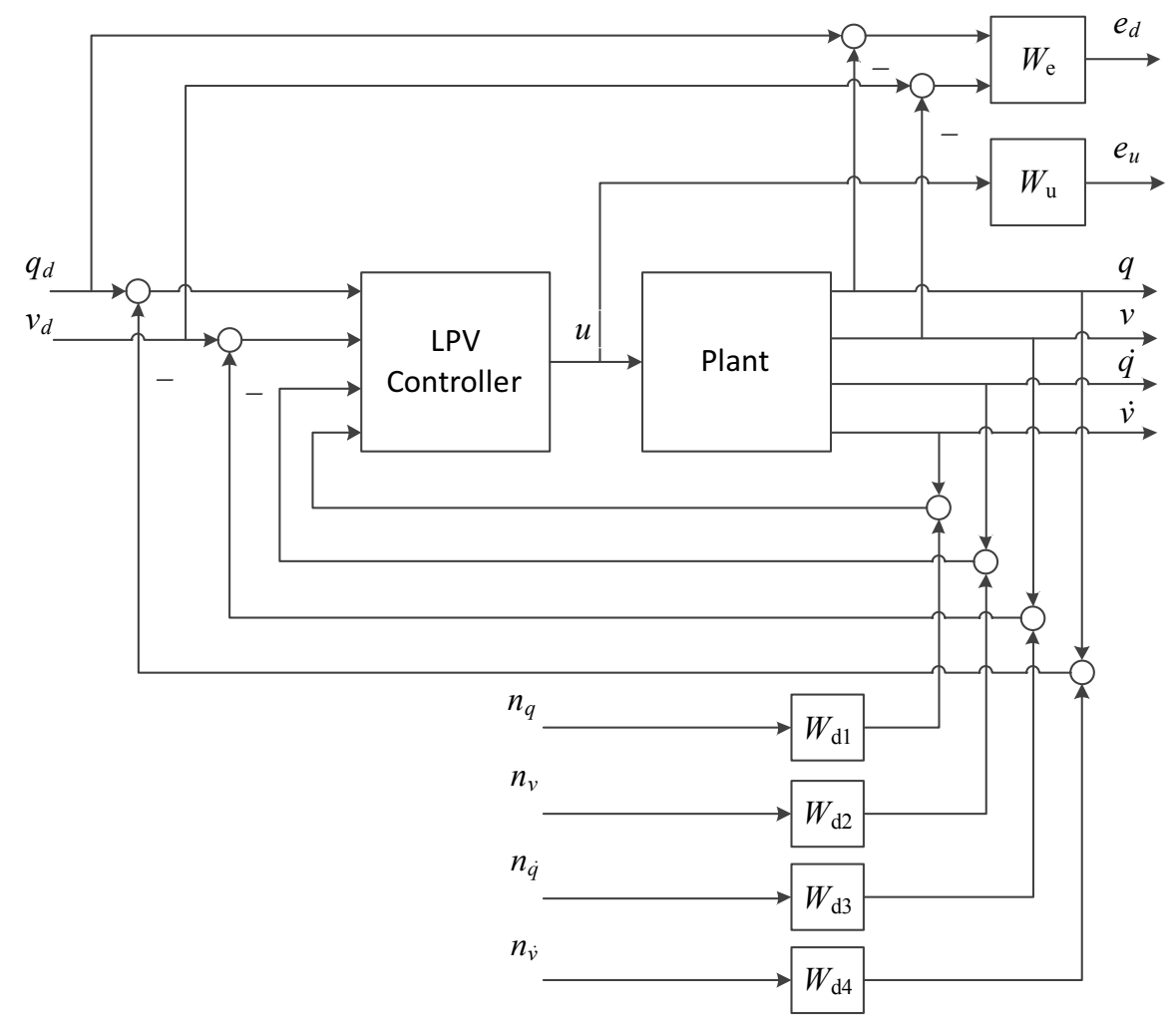

Fig. 5 System interconnections

According to the system interconnections shown in Fig. 5, the generalized input vector $\boldsymbol{d}$ can be written as: $\boldsymbol{d}=$ $\left[\boldsymbol{q}_{\boldsymbol{d}}{ }^{T} \boldsymbol{v}_{\boldsymbol{d}}{ }^{T} \boldsymbol{n}_{\boldsymbol{q}}{ }^{T} \boldsymbol{n}_{\boldsymbol{v}}{ }^{T} \boldsymbol{n}_{\dot{q}}{ }^{T} \boldsymbol{n}_{\dot{\boldsymbol{v}}}{ }^{T}\right]^{T}$, where $\boldsymbol{q}_{\boldsymbol{d}}$ and $\boldsymbol{v}_{\boldsymbol{d}}$ are the reference signals for $\boldsymbol{q}$ and $\boldsymbol{v}$, respectively. Here $\boldsymbol{n}_{\boldsymbol{q}}, \boldsymbol{n}_{\boldsymbol{v}}, \boldsymbol{n}_{\dot{q}}$, and $\boldsymbol{n}_{\dot{\boldsymbol{v}}}$ represent the measurement noise and disturbances. The generalized error vector $\boldsymbol{e}$ can be expressed as: $\boldsymbol{e}=\left[\boldsymbol{e}_{\boldsymbol{d}}{ }^{T}\right.$ $\left.\boldsymbol{e}_{u}^{T}\right]^{T}$. Note that membrane deflections and membrane deflection rates are included into the feedback signals. Laser vibrometers can be used to acquire these data in practice. More details can be found in, for example, [10] and [66].

Matrices $\boldsymbol{R}(\rho(t)), \boldsymbol{S}(\rho(t)), \overline{\boldsymbol{A}}_{\boldsymbol{k}}(\rho(t)), \overline{\boldsymbol{B}}_{\boldsymbol{k}}(\rho(t)), \overline{\boldsymbol{C}}_{\boldsymbol{k}}(\rho(t))$, and $\boldsymbol{D}_{\boldsymbol{k}}(\rho(t))$ in (31) are parameterized as:

$$
\begin{array}{ll}
\boldsymbol{R}(\rho(t))=\boldsymbol{R}_{\mathbf{0}}+\rho_{1} \boldsymbol{R}_{1}+\rho_{2} \boldsymbol{R}_{2} ; & \boldsymbol{S}(\rho(t))=\boldsymbol{S}_{\mathbf{0}} \\
\overline{\boldsymbol{A}}_{\boldsymbol{k}}(\rho(t))=\overline{\boldsymbol{A}}_{\boldsymbol{k} 0} ; & \overline{\boldsymbol{B}}_{\boldsymbol{k}}(\rho(t))=\overline{\boldsymbol{B}}_{\boldsymbol{k} \mathbf{0}} \\
\overline{\boldsymbol{C}}_{\boldsymbol{k}}(\rho(t))=\overline{\boldsymbol{C}}_{\boldsymbol{k} \mathbf{0}} ; & \boldsymbol{D}_{\boldsymbol{k}}(\rho(t))=\boldsymbol{D}_{\boldsymbol{k} \mathbf{0}}
\end{array}
$$

Note that $\boldsymbol{R}_{\mathbf{0}}, \boldsymbol{R}_{\mathbf{1}}, \boldsymbol{R}_{\mathbf{2}}$, and $\boldsymbol{S}_{\mathbf{0}}$ in (34) are symmetric matrices. Matrices $\boldsymbol{M}(\rho(t))$ and $\boldsymbol{N}(\rho(t))$ are chosen as:

$$
\boldsymbol{M}(\rho(t))=\boldsymbol{I}-\boldsymbol{R}(\rho(t)) \boldsymbol{S}(\rho(t)) ; \quad \boldsymbol{N}(\rho(t))=\boldsymbol{I}
$$

The bounds for the rates of $\rho_{1}$ and $\rho_{2}$ are set to be $\pm 10 \mathrm{deg} / \mathrm{s}$, i.e. 


$$
\begin{array}{lll}
\underline{v}_{1} \leq \dot{\rho}_{1} \leq \bar{v}_{1} ; & \left(\bar{v}_{1}=10 \mathrm{deg} / \mathrm{s} ;\right. & \left.\underline{v}_{1}=-10 \mathrm{deg} / \mathrm{s}\right) \\
\underline{v}_{2} \leq \dot{\rho}_{2} \leq \bar{v}_{2} ; & \left(\bar{v}_{2}=10 \mathrm{deg} / \mathrm{s} ; \quad \underline{v}_{2}=-10 \mathrm{deg} / \mathrm{s}\right)
\end{array}
$$

According to the gridding approach introduced in [29] and the matrix parameterization in (34) and (35), solving the convex optimization problem expressed in $(31)$ on $\mathcal{P}_{\mathcal{D}}$ is equivalent to solving the following problem on $\mathcal{P}_{\mathcal{D}}$ :

$$
\begin{array}{ll}
\text { find } & \boldsymbol{R}_{0}, \boldsymbol{R}_{1}, \boldsymbol{R}_{2}, \boldsymbol{S}_{0}, \bar{A}_{k 0}, \overline{\boldsymbol{B}}_{k 0}, \overline{\boldsymbol{C}}_{k 0}, \text { and } \boldsymbol{D}_{k 0} \\
\text { subject to } & \boldsymbol{L} \boldsymbol{M}_{1} \succ \boldsymbol{O} \\
& \boldsymbol{L} \boldsymbol{M}_{2} \prec \boldsymbol{O} \\
& \boldsymbol{L} \boldsymbol{M}_{3} \prec \boldsymbol{O} \\
& \boldsymbol{L} \boldsymbol{M}_{4} \prec \boldsymbol{O} \\
& \boldsymbol{L} \boldsymbol{M}_{5} \prec \boldsymbol{O}
\end{array}
$$

where

$$
\begin{aligned}
& \boldsymbol{L} \boldsymbol{M}_{\mathbf{1}}=\left[\begin{array}{cc}
\boldsymbol{R}_{\mathbf{0}}+\rho_{1} \boldsymbol{R}_{\mathbf{1}}+\rho_{2} \boldsymbol{R}_{\mathbf{2}} & \boldsymbol{I} \\
\boldsymbol{I} & \boldsymbol{S}_{\mathbf{0}}
\end{array}\right] \\
& \boldsymbol{L} \boldsymbol{M}_{2}=\left[\begin{array}{ccc}
\dot{\Psi}_{11}+\Psi_{\mathrm{A}}+\Psi_{\mathrm{A}}{ }^{T} & \Psi_{\mathrm{B}} & \Psi_{\mathrm{C}}{ }^{T} \\
\Psi_{\mathrm{B}}{ }^{T} & -\gamma \boldsymbol{I} & \Psi_{\mathrm{D}}{ }^{T} \\
\Psi_{\mathrm{C}} & \Psi_{\mathrm{D}} & -\gamma \boldsymbol{I}
\end{array}\right] ; \quad \boldsymbol{L} \boldsymbol{M}_{\mathbf{3}}=\left[\begin{array}{ccc}
\dot{\Psi}_{12}+\Psi_{\mathrm{A}}+\Psi_{\mathrm{A}}{ }^{T} & \Psi_{\mathrm{B}} & \Psi_{\mathrm{C}}{ }^{T} \\
\Psi_{\mathrm{B}}{ }^{T} & -\gamma \boldsymbol{I} & \Psi_{\mathbf{D}}{ }^{T} \\
\Psi_{\mathrm{C}} & \Psi_{\mathbf{D}} & -\gamma \boldsymbol{I}
\end{array}\right] \\
& \boldsymbol{L} \boldsymbol{M}_{4}=\left[\begin{array}{ccc}
\dot{\Psi}_{21}+\Psi_{\mathrm{A}}+\Psi_{\mathrm{A}}{ }^{T} & \Psi_{\mathrm{B}} & \boldsymbol{\Psi}_{\mathrm{C}}{ }^{T} \\
\Psi_{\mathbf{B}}{ }^{T} & -\gamma \boldsymbol{I} & \Psi_{\mathbf{D}}{ }^{T} \\
\Psi_{\mathrm{C}} & \Psi_{\mathbf{D}} & -\gamma \boldsymbol{I}
\end{array}\right] ; \quad \boldsymbol{L} \boldsymbol{M}_{\mathbf{5}}=\left[\begin{array}{ccc}
\dot{\Psi}_{22}+\Psi_{\mathrm{A}}+\Psi_{\mathrm{A}}{ }^{T} & \Psi_{\mathrm{B}} & \Psi_{\mathrm{C}^{T}} \\
\Psi_{\mathbf{B}}{ }^{T} & -\gamma \boldsymbol{I} & \Psi_{\mathbf{D}}{ }^{T} \\
\Psi_{\mathrm{C}} & \Psi_{\mathbf{D}} & -\gamma \boldsymbol{I}
\end{array}\right]
\end{aligned}
$$

Matrices $\dot{\Psi}_{11}, \dot{\Psi}_{12}, \dot{\Psi}_{21}$, and $\dot{\Psi}_{22}$ in (38) are defined as:

$$
\begin{array}{ll}
\dot{\Psi}_{11}=\left[\begin{array}{cc}
\bar{v}_{1} \boldsymbol{R}_{1}+\bar{v}_{2} \boldsymbol{R}_{2} & \boldsymbol{O} \\
\boldsymbol{O} & \boldsymbol{O}
\end{array}\right] ; & \dot{\Psi}_{12}=\left[\begin{array}{cc}
\bar{v}_{1} \boldsymbol{R}_{1}+\underline{v}_{2} \boldsymbol{R}_{2} & \boldsymbol{O} \\
\boldsymbol{O} & \boldsymbol{O}
\end{array}\right] \\
\dot{\Psi}_{21}=\left[\begin{array}{cc}
\underline{v}_{1} \boldsymbol{R}_{1}+\bar{v}_{2} \boldsymbol{R}_{2} & \boldsymbol{O} \\
\boldsymbol{O} & \boldsymbol{O}
\end{array}\right] ; & \dot{\Psi}_{22}=\left[\begin{array}{cc}
v_{1} \boldsymbol{R}_{1}+\underline{v}_{2} \boldsymbol{R}_{2} & \boldsymbol{O} \\
\boldsymbol{O} & O
\end{array}\right]
\end{array}
$$

According to the approaches introduced in [35-37], the weighting functions used in this work are selected as follows:

1. To specify the tracking error performance, the weighting function $\boldsymbol{W}_{\mathrm{e}}$ is defined, in frequency domain, as:

$$
\boldsymbol{W}_{\boldsymbol{e}}=\operatorname{diag}\left(W_{e 1}(s) \boldsymbol{I}_{21 \times 21} \quad W_{e 2}(s) \boldsymbol{I}_{4 \times 4}\right)
$$

where $s$ is a complex variable for a transfer function and

$$
W_{e 1}(s)=\frac{0.1 s+30}{s+0.3} ; \quad W_{e 2}(s)=\frac{0.05 s+50}{s+2.5}
$$


The weighting function $W_{e 1}(s)$ is used to specify the tracking performance of $\boldsymbol{q}$. The gain of $W_{e 1}(s)$ at low frequencies is: $W_{e 1}(0)=100$, corresponding to the desired steady-state error: $e_{\infty}=1 / W_{e 1}(0)=1 \%$. Similarly, the tracking performance of $\boldsymbol{\eta}$ can be determined by the weighting function $W_{e 2}(s)$, whose gain at low frequencies is: $W_{e 2}(0)=20$, indicating that the desired steady-state error of $\eta$ is: $e_{\infty}=1 / W_{e 2}(0)=5 \%$.

2. The weighting function $W_{\mathrm{u}}$ is selected to prevent the tendons from going slack during simulations. It is desired that the tendon variations to unit step inputs are less than $0.05 \mathrm{~m}$, which gives the weight: $\boldsymbol{W}_{\mathbf{u}}=1 / 0.05$ $\boldsymbol{I}_{21 \times 21}=20 \boldsymbol{I}_{21 \times 21}$.

3. The weighting functions corresponding to the measurement noise are chosen as: $\boldsymbol{W}_{\mathrm{d} 1}=0.001 \boldsymbol{I}_{21 \times 21}, \boldsymbol{W}_{\mathrm{d} 2}=$ $0.001 \boldsymbol{I}_{4 \times 4}, \boldsymbol{W}_{\mathrm{d} 3}=0.001 \boldsymbol{I}_{21 \times 21}$, and $\boldsymbol{W}_{\mathrm{d} 4}=0.001 \boldsymbol{I}_{4 \times 4}$. These weighting functions indicate that the sensor noises for locations, velocities, angles, angle rates, membrane deflections, and membrane deflection rates are modeled as white noises with maximum amplitudes of $1 \mathrm{~mm}, 1 \mathrm{~mm} / \mathrm{s}, 0.06 \mathrm{deg}, 0.06 \mathrm{deg} / \mathrm{s}, 1 \mathrm{~mm}$, and $1 \mathrm{~mm} / \mathrm{s}$, respectively.

\section{Simulation Results}

In this section, the LPV controller designed in Section 5 is used to deploy a 2-3 tensegrity-membrane system from its initial packaged configuration to its final deployed configuration. The nonlinear finite element model and the control-oriented model are used in numerical simulations. The materials of membranes, bars, and tendons are chosen as Kapton, aluminum, and Nylon, respectively. The parameters of system components are listed in Table 1.

Table 1. Material and dimensional parameters of the tensegrity-membrane systems

\begin{tabular}{cccc}
\hline Parameters & Membrane & Bar & Tendon \\
\hline Young's modulus & $165 \mathrm{MPa}$ & $69 \mathrm{GPa}$ & $2 \mathrm{GPa}$ \\
Density & $1400 \mathrm{~kg} / \mathrm{m}^{3}$ & $2700 \mathrm{~kg} / \mathrm{m}^{3}$ & N/A \\
Poisson's ratio & 0.34 & N/A & N/A \\
\hline
\end{tabular}

As mentioned in the modeling assumptions presented in Section 3, for the two models used in this work, tendon dynamics is neglected and the effect of tendons on a tensegrity-membrane system is modeled as tendon forces due to 
longitudinal elongations. Therefore, tendon density and Poisson's ratio are not specified in Table 1. Also, the bars are modeled as struts that experience longitudinal deformations for the nonlinear finite element model, and they are treated as rigid bodies for the control-oriented model. Clearly, Poisson's ratio for each bar is not needed for these two models and is not specified in Table 1.

The membrane is in the shape of an equilateral triangle, and the radius of the circumscribed circle for this triangle is $1 \mathrm{~m}$. The thickness of the membrane is chosen as $51 \mu \mathrm{m}$. Bars with circular cross-sections are used. The bar length is chosen as $1.8 \mathrm{~m}$, i.e. $l_{i j}=l=1.8 \mathrm{~m}$, and the diameter of the cross-section is chosen as $30 \mathrm{~mm}$. Each tendon has a circular cross-section. The diameters of tendon cross-sections are listed in Table 2.

\section{Table 2. Diameters of tendon cross-sections}

\begin{tabular}{ll}
\hline Tendon & Diameter \\
\hline Tendon $S_{1 j}$ & $0.2 \mathrm{~mm}$ \\
Tendon $V_{1 j}$ & $0.254 \mathrm{~mm}$ \\
Tendon $D_{1 j}$ & $0.3 \mathrm{~mm}$ \\
Tendon $C_{1 j}$ & $0.4 \mathrm{~mm}$ \\
Tendon $P_{1 j}$ & $0.16 \mathrm{~mm}$ \\
Tendon $R_{1 j}$ & $0.2 \mathrm{~mm}$ \\
Tendon $S_{2 j}$ & $0.16 \mathrm{~mm}$ \\
\hline
\end{tabular}

As discussed previously, $\theta$ and $\psi$ can specify symmetric equilibrium configurations. Thus, once the reference angles, $\theta_{r}$ and $\psi_{r}$, are specified, the reference signal $\boldsymbol{q}_{\boldsymbol{d}}$ can be determined according to (2) by letting $\theta=\theta_{r}$ and $\psi=$ $\psi_{r}$. In this work, two desired trajectories for reference angles are used. For the first desired trajectory, $\psi_{r}$ is constant and $\theta_{r}$ is generated by a second-order system:

$$
\begin{aligned}
& \ddot{\theta}_{r}(t)+2 \xi_{r} \omega_{r} \dot{\theta}_{r}(t)+\omega_{r}^{2} \theta_{r}(t)=\omega_{r}^{2} \theta_{e} ; \quad\left(\theta_{r}\left(t_{0}\right)=\theta_{0} ; \quad \dot{\theta}_{r}\left(t_{0}\right)=0\right) \\
& \psi_{r}(t)=\psi_{0}
\end{aligned}
$$

The subscripts 0 and $e$ represent the values of the initial and deployed (erected) configurations, respectively. This trajectory is referred to as "Trajectory A". The major advantage of this desired trajectory is that the initial and final 
values of $\ddot{\theta}_{r}$ are zero (i.e. $\ddot{\theta}_{r}\left(t_{0}\right)=0$ and $\left.\ddot{\theta}_{r}(\infty)=0\right)$, which is expected to make tensegrity-membrane systems experience smooth acceleration and deceleration during system deployment.

For the second desired trajectory, $\psi_{r}$ is constant and $\theta_{r}$ is generated by the following $C^{0}$ function:

$$
\begin{aligned}
& \theta_{r}(t)= \begin{cases}\theta_{0} ; & t \leq t_{1} \\
\theta_{0}+\frac{t-t_{1}}{t_{2}-t_{1}}\left(\theta_{e}-\theta_{0}\right) ; & t_{1}<t \leq t_{2} \\
\theta_{e} ; & t>t_{2}\end{cases} \\
& \psi_{r}(t)=\psi_{0}
\end{aligned}
$$

where $t_{1}$ and $t_{2}$ are time instants. This trajectory is referred to as "Trajectory B". Clearly, this trajectory is much easier to implement in practice compared to Trajectory A, since the generation of Trajectory A requires solving a second-order system during system deployment, leading to higher requirement for the hardware of the controller. However, discontinuities in the time derivatives of $\theta_{r}$ at $t=t_{1}$ and $t=t_{2}$ may introduce large impacts (e.g., forces) on the system components and cause large amplitude vibrations of the membrane.

The system initial configuration for deployment is chosen as a system equilibrium determined by the formfinding method for tensegrity-membrane systems proposed in our previous work [25]. According to the parameters of the initial configuration, initialization steps before conducting numerical simulations are needed for the nonlinear finite element model. The system reference configuration (i.e. the system configuration at time 0 mentioned in Section 3.1), where the membrane and bars are undeformed, is chosen in the vicinity of the equilibrium corresponding to the initial configuration. The dynamic relaxation technique is used to drive the system from the reference configuration to the initial configuration under the effect of artificial damping. This initialization approach for the nonlinear finite element model was also used in our previous work [25], where more details can be found.

For the two models, Park's method [32] is used to solve the corresponding equations of motion. If $\tilde{\boldsymbol{x}}$ is the generalized coordinate vector, the velocity and acceleration terms can be expressed as:

$$
\begin{aligned}
& \ddot{\tilde{\boldsymbol{x}}}_{t+\Delta t}=\frac{1}{6 \Delta t}\left(10 \dot{\tilde{\boldsymbol{x}}}_{t+\Delta t}-15 \dot{\tilde{\boldsymbol{x}}}_{t}+6 \dot{\tilde{\boldsymbol{x}}}_{t-\Delta t}-\dot{\tilde{\boldsymbol{x}}}_{t-2 \Delta t}\right) \\
& \dot{\tilde{\boldsymbol{x}}}_{t+\Delta t}=\frac{1}{6 \Delta t}\left(10 \tilde{\boldsymbol{x}}_{t+\Delta t}-15 \tilde{\boldsymbol{x}}_{t}+6 \tilde{\boldsymbol{x}}_{t-\Delta t}-\tilde{\boldsymbol{x}}_{t-2 \Delta t}\right)
\end{aligned}
$$

Since Park's method is a 3-step method, initialization is required. The trapezoidal rule is applied for the initialization process: 


$$
\tilde{\boldsymbol{x}}_{t+\Delta t}=\tilde{\boldsymbol{x}}_{t}+\frac{\Delta t}{2}\left(\dot{\tilde{\boldsymbol{x}}}_{t}+\dot{\tilde{\boldsymbol{x}}}_{t+\Delta t}\right) ; \quad \dot{\tilde{\boldsymbol{x}}}_{t+\Delta t}=\dot{\tilde{\boldsymbol{x}}}_{t}+\frac{\Delta t}{2}\left(\ddot{\tilde{\boldsymbol{x}}}_{t}+\ddot{\tilde{\boldsymbol{x}}}_{t+\Delta t}\right)
$$

\subsection{LPV Controller Verification}

Since a gridding method and a $5 \times 3$ grid is used to reduce the infinite-dimensional LMI problem in (31) to a finite-dimensional problem shown in (37), it is necessary to verify that the resulting control parameters can satisfy the LMIs in (37) in the parameter space $\mathcal{P}$. Usually, grids that are denser than the control design grid are used to discretize $\mathcal{P}$ in order to conduct the verification. In this work, a $21 \times 7$ verification grid, plotted in Fig. 4 , is used to conduct the verification for the LPV controller discussed in Section 5. The minimum eigenvalue of $\boldsymbol{L M}_{\mathbf{1}}$ (i.e. $\left.\lambda_{\min }\left(\boldsymbol{L} \boldsymbol{M}_{1}\right)\right)$ and the maximum eigenvalues of $\boldsymbol{L} \boldsymbol{M}_{\mathbf{2}}, \boldsymbol{L} \boldsymbol{M}_{\mathbf{3}}, \boldsymbol{L} \boldsymbol{M}_{\mathbf{4}}, \boldsymbol{L} \boldsymbol{M}_{\mathbf{5}}\left(\right.$ i.e. $\left.\lambda_{\max }\left(\boldsymbol{L} \boldsymbol{M}_{i}\right),(i=2, \ldots, 5)\right)$ are calculated

over this verification grid. The numerical results are plotted in Fig. 6. It can be seen that $\lambda_{\min }\left(\boldsymbol{L} \boldsymbol{M}_{\mathbf{1}}\right)>0$ and $\lambda_{\max }\left(\boldsymbol{L} \boldsymbol{M}_{i}\right)<0(i=2, \ldots, 5)$ over the verification grid, indicating that the solution of the LPV controller satisfies the LMIs in (37) on this much finer grid in the parameter space $\mathcal{P}$.
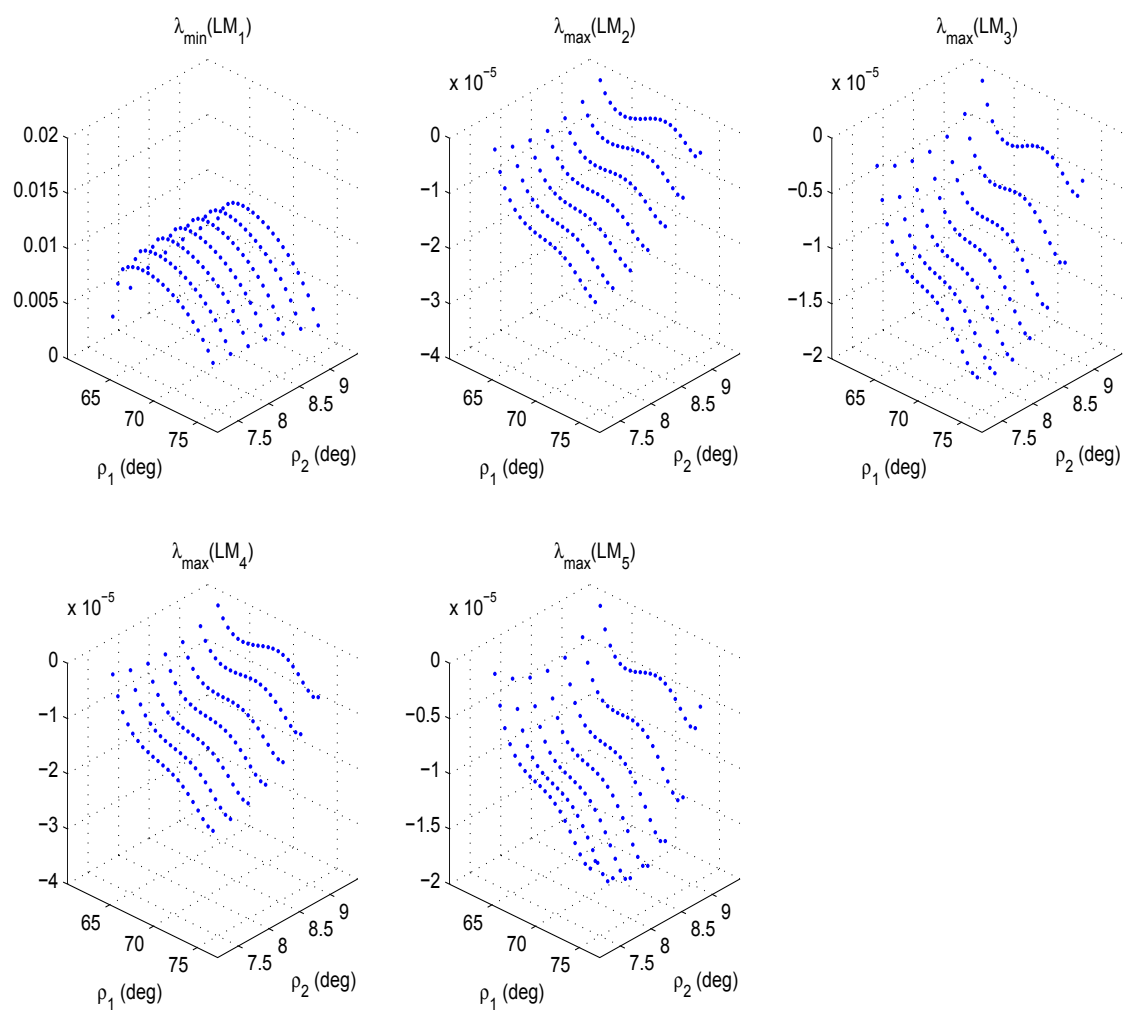


\section{Fig. 6. Results of LPV controller verification}

\subsection{System Deployment}

In this section, the nonlinear finite element model and the control-oriented model are used in numerical simulations to test the performance of the LPV controller in deploying a 2-3 tensegrity-membrane system. Membrane vibrations are studied by monitoring the transverse deflections of four points on the membrane, which are labeled $w_{1}, w_{2}, w_{3}$, and $w_{4}$. These transverse deflections are measured in the $O_{f}-x_{f} y_{f} z_{f}$ frame and are characterized by:

$$
\begin{aligned}
& w_{1}=\left.w\left(x_{f}, y_{f}\right)\right|_{x_{f}=0.86(\mathrm{~m}) ; y_{f}=0.50(\mathrm{~m})} \\
& w_{2}=\left.w\left(x_{f}, y_{f}\right)\right|_{x_{f}=1.30(\mathrm{~m}) ; y_{f}=0.75(\mathrm{~m})} \\
& w_{3}=\left.w\left(x_{f}, y_{f}\right)\right|_{x_{f}=0.43(\mathrm{~m}) ; y_{f}=0.75(\mathrm{~m})} \\
& w_{4}=\left.w\left(x_{f}, y_{f}\right)\right|_{x_{f}=0.87(\mathrm{~m}) ; y_{f}=0(\mathrm{~m})}
\end{aligned}
$$

The time step is chosen as $\Delta t=0.01 \mathrm{~s}$ according to Shannon's sampling theorem [33] and the highest natural frequency of the closed-loop system given by the control-oriented model. The highest natural frequency of the closed-loop system is $31.85 \mathrm{~Hz}$. The sampling frequency is chosen to be approximately 4 times higher than 31.85 $\mathrm{Hz}$, which gives the corresponding time step $\Delta t=0.01 \mathrm{~s}$. In order to perform fair comparisons, the same time step is used to solve the nonlinear finite element model as well.

\subsubsection{Trajectory $A$}

The equations in (42) are used to generate the desired trajectories. The parameters used in this case are: $\xi_{r}=1, \omega_{r}$ $=0.28, \theta_{0}=74.46^{\circ}, \theta_{e}=63^{\circ}$, and $\psi_{0}=8.36^{\circ}$. Clearly, the value of $\theta_{r}$ is generated by a critically damped secondorder system with the settling time of 16.96 seconds.

Simulation results are shown in Fig. 7 - Fig. 10. We can observe that the LPV controller can regulate the behavior of bars during deployment. The responses of the tensegrity-membrane system are very close to the desired trajectory and the tracking error is small. Transverse deflections of the four points on the membrane, $w_{1}, w_{2}, w_{3}$, and $w_{4}$, are shown in Fig. 7. It can be seen that the LPV controller can also regulate the behavior of the membrane. There is no significant vibration introduced during system deployment and the maximum deflection is less than $0.5 \mathrm{~mm}$. From the results shown in Fig. 8, we conclude that tendons are always in tension during system deployment, since 
tendon rest-lengths are smaller than control limits, which are the corresponding tendon lengths. It can be also seen that the simulation results given by the two models are in good agreement, indicating that the control-oriented model has sufficient accuracy for control design and can provide accurate system responses in simulations when appropriate controls are applied.
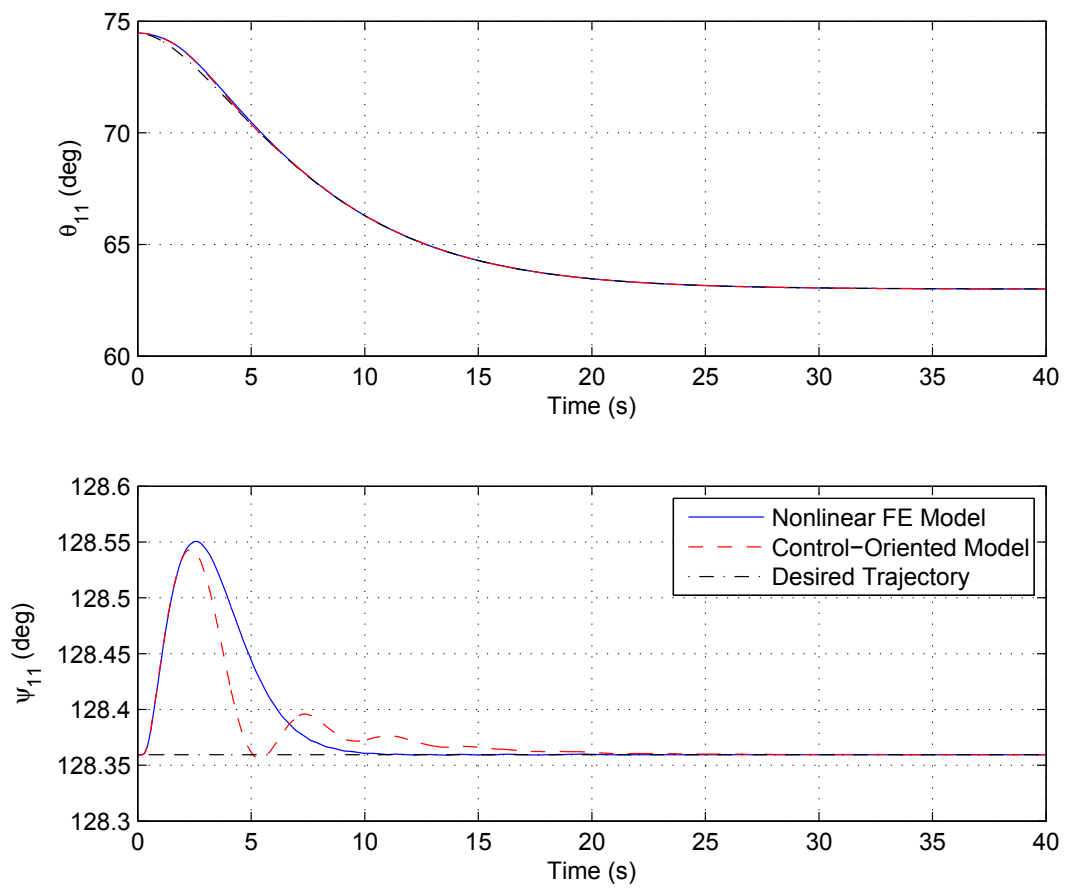

Fig. 7 Responses of bar $b_{11}$ (Trajectory A) 

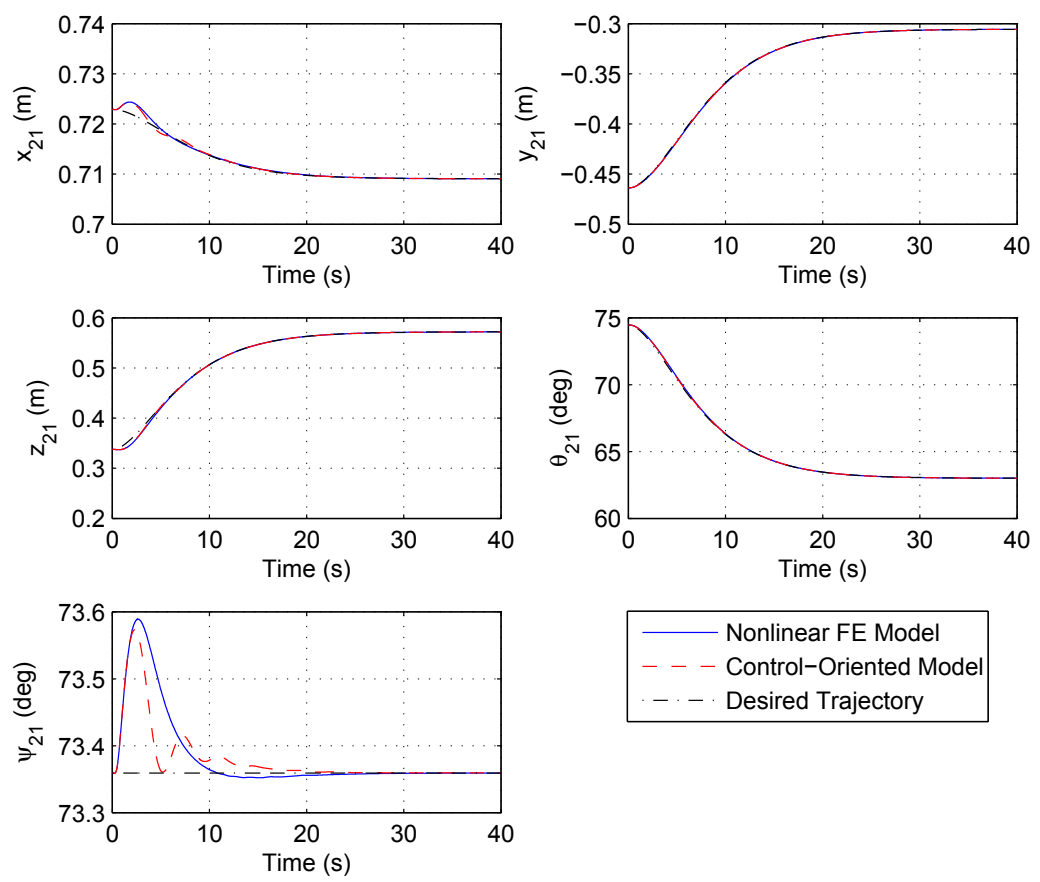

Fig. 8 Responses of bar $b_{21}$ (Trajectory A)
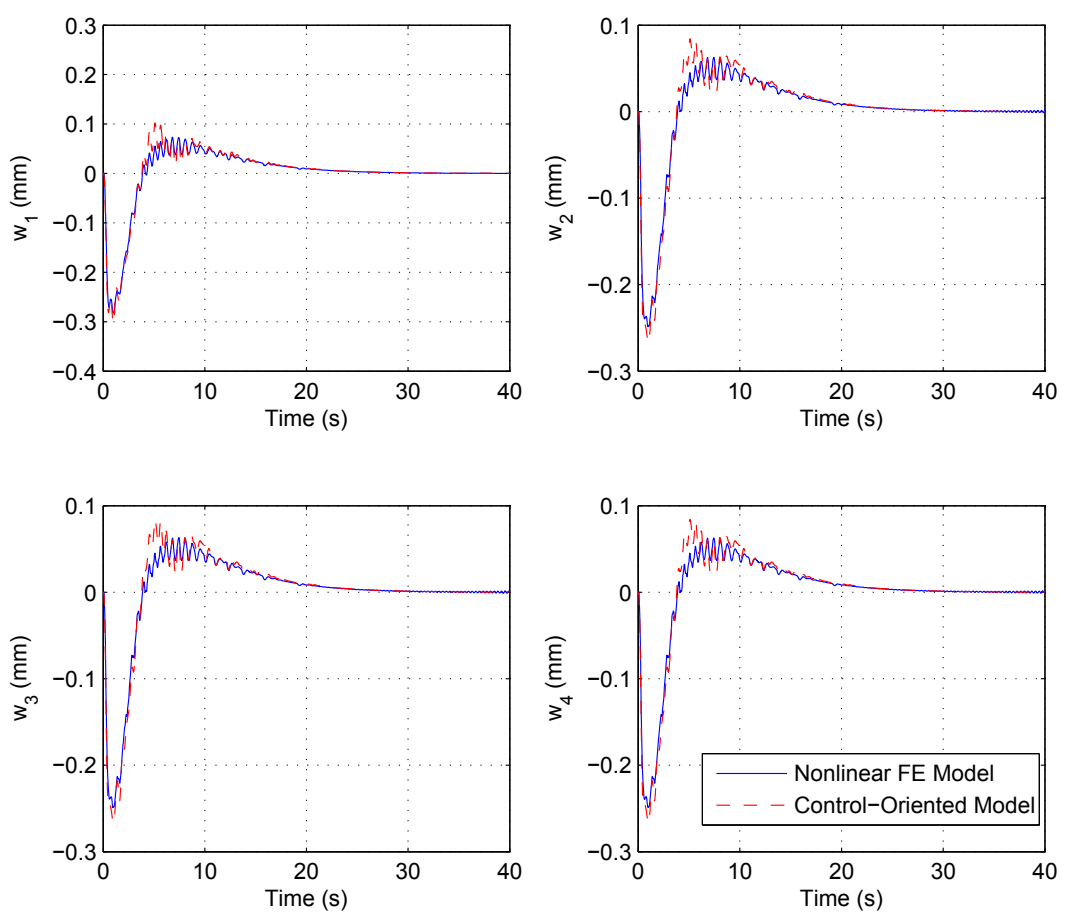

Fig. 9 Transverse deflections of the membrane in the $O_{f}-x_{f} y_{f} z_{f}$ frame (Trajectory A) 

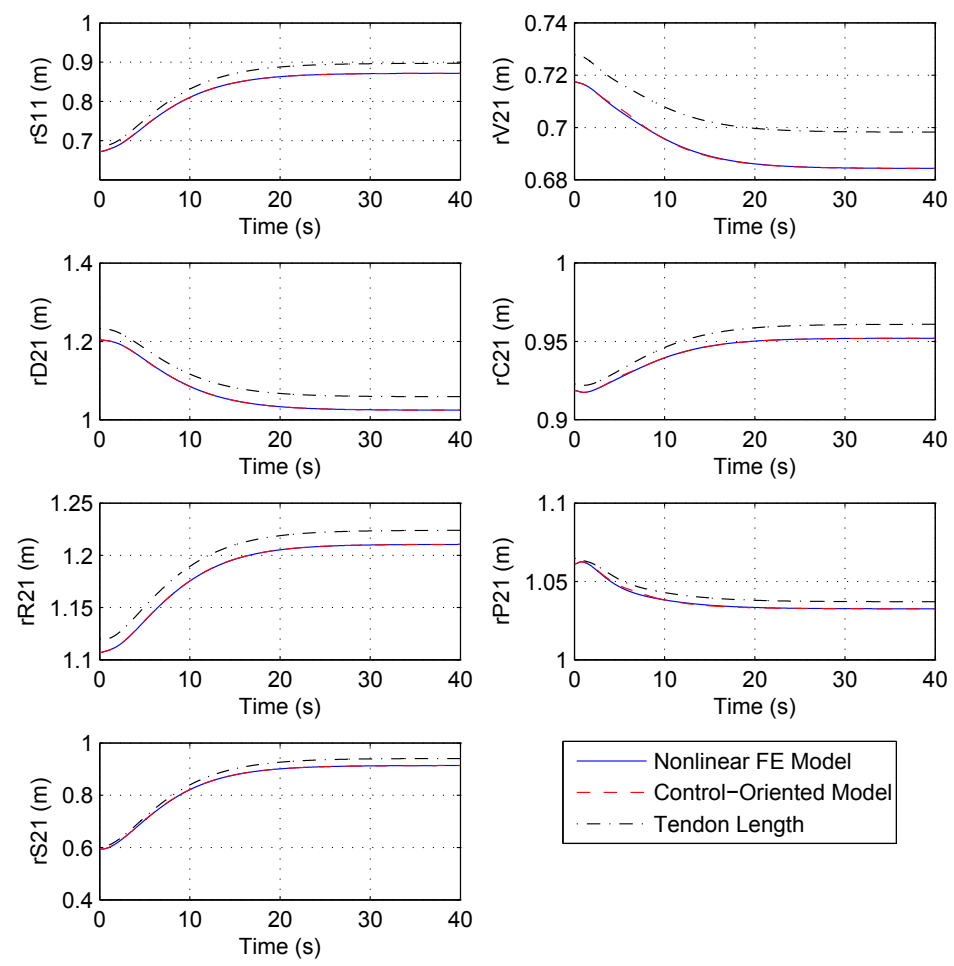

Fig. 10 Representative tendon rest-length variations (Trajectory A)

Snapshots of system deployment based on the nonlinear finite element simulation results are shown in Fig. 11. It can be clearly observed that the tensegrity-membrane system experiences significant shape changes, while the membrane transverse deflections can hardly be seen. This result confirms the simulation results in Fig. 9, where the maximum membrane deflection is less than $0.5 \mathrm{~mm}$.

System deployment lasts for 40 seconds. According to Figs. 7-10, since the desired trajectory is generated by a second-order system, the rate of system deployment slows down at the end of deployment in order to introduce less deceleration to system components. This result can be also observed in Fig. 11, since there is no significant difference in system height according to system configurations at $t=10 \mathrm{~s}$ and $t=40 \mathrm{~s}$, indicating that the rate of system deployment is small when $t>10$ s. 


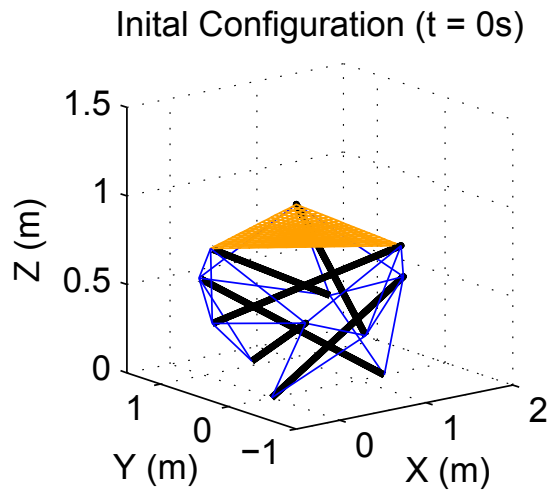

Intermediate Configuration $(t=10 \mathrm{~s})$

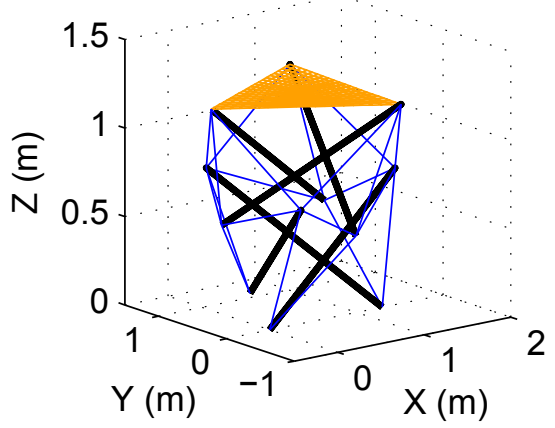

\section{Intermediate Configuration $(t=5 s)$}

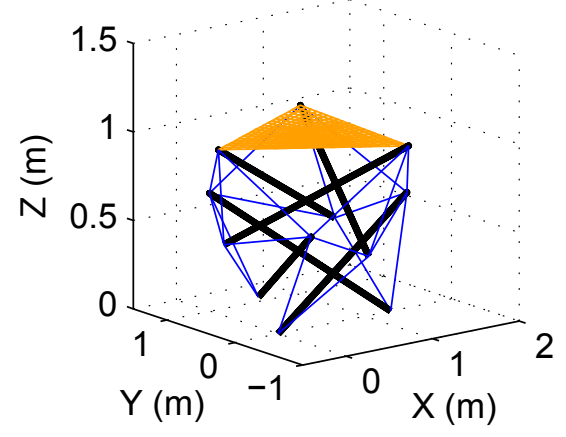

Final Configuration $(t=40 s)$

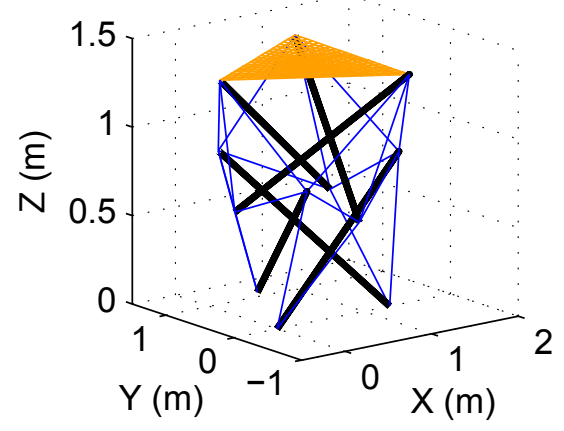

Fig. 11 Deployment sequence of a tensegrity-membrane system (Trajectory A)

Since the bounds of parameter varying rates are considered in the LPV control design, it is necessary to check whether these parameter rate constraints are satisfied during simulations. As discussed in Section 5, the bounds for the rates of $\theta_{13}$ (i.e. $\rho_{1}$ ) and $\psi_{13}$ (i.e. $\rho_{2}$ ) are chosen as $10 \mathrm{deg} / \mathrm{s}$. To examine the satisfaction of parameter rate constraints, $\dot{\theta}_{13}$ and $\dot{\psi}_{13}$ are monitored during system deployment. The peak values of $\left|\dot{\theta}_{13}\right|$ given by the nonlinear finite element model and the control-oriented model are $1.144 \mathrm{deg} / \mathrm{s}$ and $1.186 \mathrm{deg} / \mathrm{s}$, respectively. The peak values of $\left|\dot{\psi}_{13}\right|$ given by the nonlinear finite element model and the control-oriented model are $0.142 \mathrm{deg} / \mathrm{s}$ and $0.144 \mathrm{deg} / \mathrm{s}$, respectively. It is obvious that the parameter varying rates are within the specified bounds and the parameter rate constraints are not violated.

The peak compressive load on bars, the maximum von Mises stress, and the maximum stress in tendons are monitored during simulations to check whether mechanical failures of system components occur during system deployment. The results given by the two models are shown in Table 3. It can be seen that the peak compressive 
load is less than the critical load determined by Euler's column buckling formula [38], the maximum von Mises stress in the membrane is less than the stress corresponding to the yield point at $3 \%$ for Kapton [39], and the maximum stress in tendons is less than the yield stress of Nylon [40]. Therefore, we can conclude that the bars do not buckle, the membrane does not yield and remains linearly elastic, and the tendons do not yield during system deployment.

Table 3. Results of yield and buckling analysis (Trajectory A)

\begin{tabular}{|c|c|c|c|c|}
\hline & $\begin{array}{l}\text { Nonlinear finite } \\
\text { element model }\end{array}$ & $\begin{array}{l}\text { Control-oriented } \\
\text { model }\end{array}$ & Critical load & Yield stress \\
\hline $\begin{array}{l}\text { Peak compressive load } \\
\text { (bar) }\end{array}$ & $6.74 \mathrm{MPa}$ & $6.74 \mathrm{~N}$ & $8357.16 \mathrm{~N}$ & N/A \\
\hline $\begin{array}{l}\text { Maximum von Mises } \\
\text { stress (membrane) }\end{array}$ & $1.13 \mathrm{MPa}$ & $1.46 \mathrm{MPa}$ & $\mathrm{N} / \mathrm{A}$ & $61.00 \mathrm{MPa}$ \\
\hline $\begin{array}{l}\text { Maximum stress } \\
\text { (tendon) }\end{array}$ & $67.08 \mathrm{MPa}$ & $67.09 \mathrm{MPa}$ & N/A & $94.80 \mathrm{MPa}$ \\
\hline
\end{tabular}

\subsubsection{Trajectory $B$}

The equations in (43) are used to generate the desired trajectories. The parameters used in this case are: $t_{1}=0.5 \mathrm{~s}$, $t_{2}=28 \mathrm{~s}, \theta_{0}=74.46^{\circ}, \theta_{e}=63^{\circ}$, and $\psi_{0}=8.36^{\circ}$. Simulation results given by the two models are shown in Fig. $12-$ Fig. 15. We can observe that the LPV controller can regulate the behavior of the system during deployment. The responses of the tensegrity-membrane system are very close to the desired trajectory and the tracking error is small.

In Fig. 13, it can be clearly seen that the attached membrane experiences large amplitude vibrations at time $t=$ $0.5 \mathrm{~s}$ and $t=28 \mathrm{~s}$, corresponding to the discontinuities of the time derivatives of $\theta_{r}$ at these two time instants specified by the desired trajectory, respectively. This result is expected, since Trajectory B, which is generated by a function that is only continuous in time (i.e. a $C^{0}$ function), results in large forces exerted on the membrane at these time instants. Compared to the results in Fig. 9, Trajectory A provides smooth acceleration and deceleration processes, so the membrane is free from significant impact due to system shape changes. Clearly, this is a major advantage of Trajectory A. 
From the results shown in Fig. 15, we can conclude that tendons are always in tension during system deployment, since tendon rest-lengths are smaller than control limits, which are the corresponding tendon lengths. It can be also seen that the simulation results given by the two models are in good agreement, indicating that the control-oriented model has sufficient accuracy for control design and can provide accurate system responses in simulations when appropriate controls are applied.
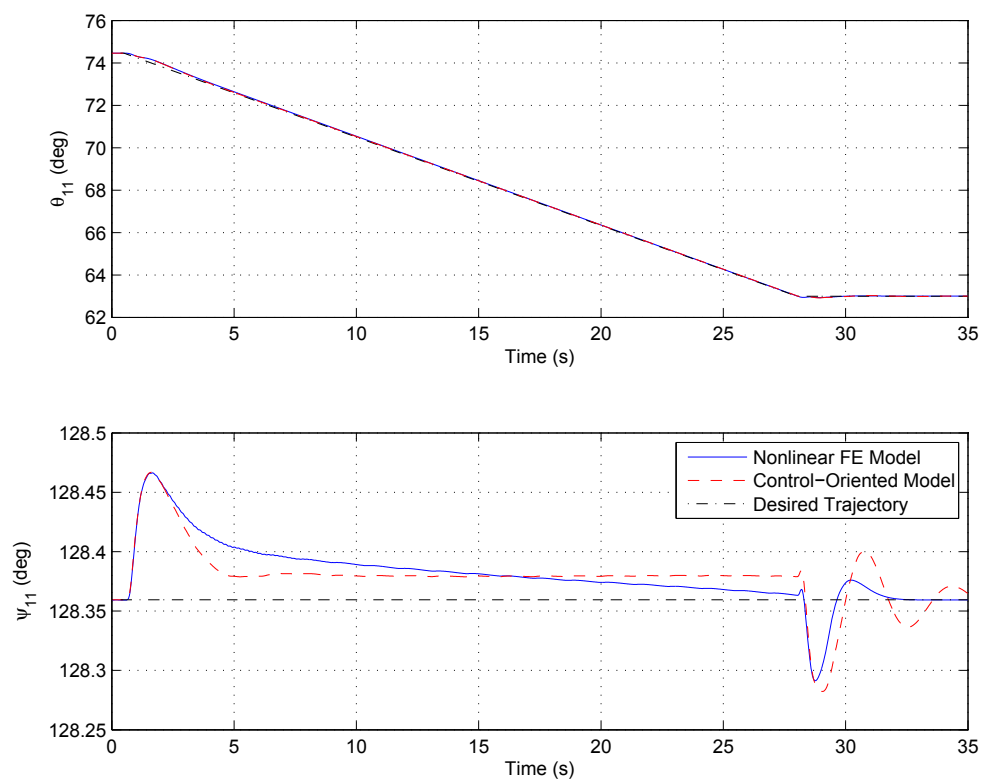

Fig. 12 Responses of bar $b_{11}$ (Trajectory B) 

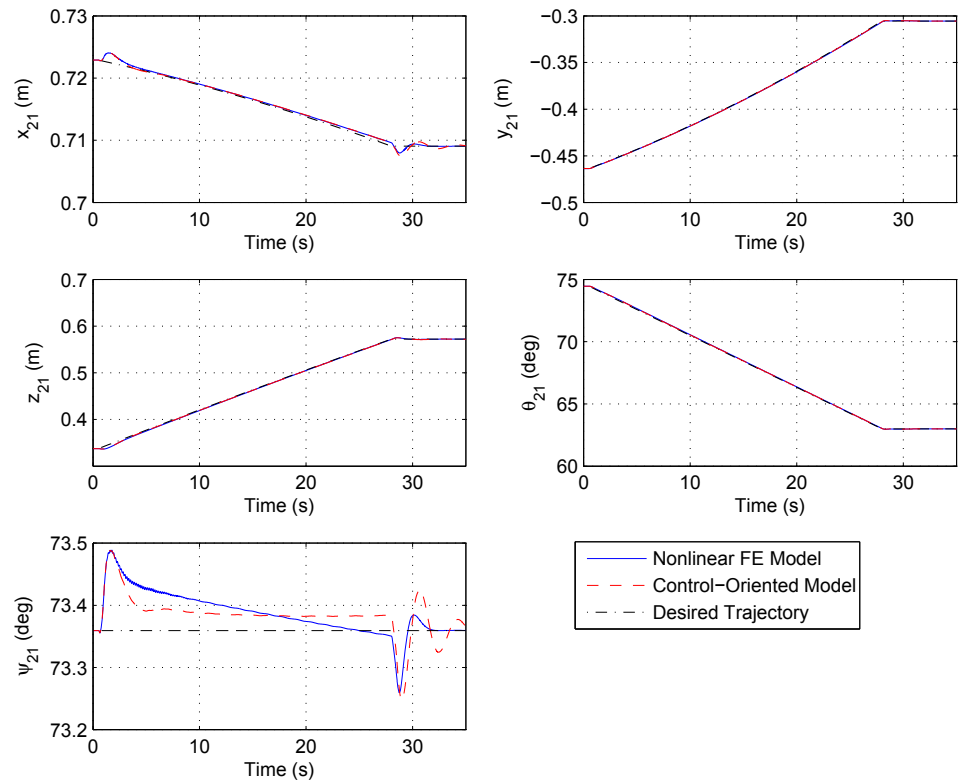

Fig. 13 Responses of bar $b_{21}$ (Trajectory B)
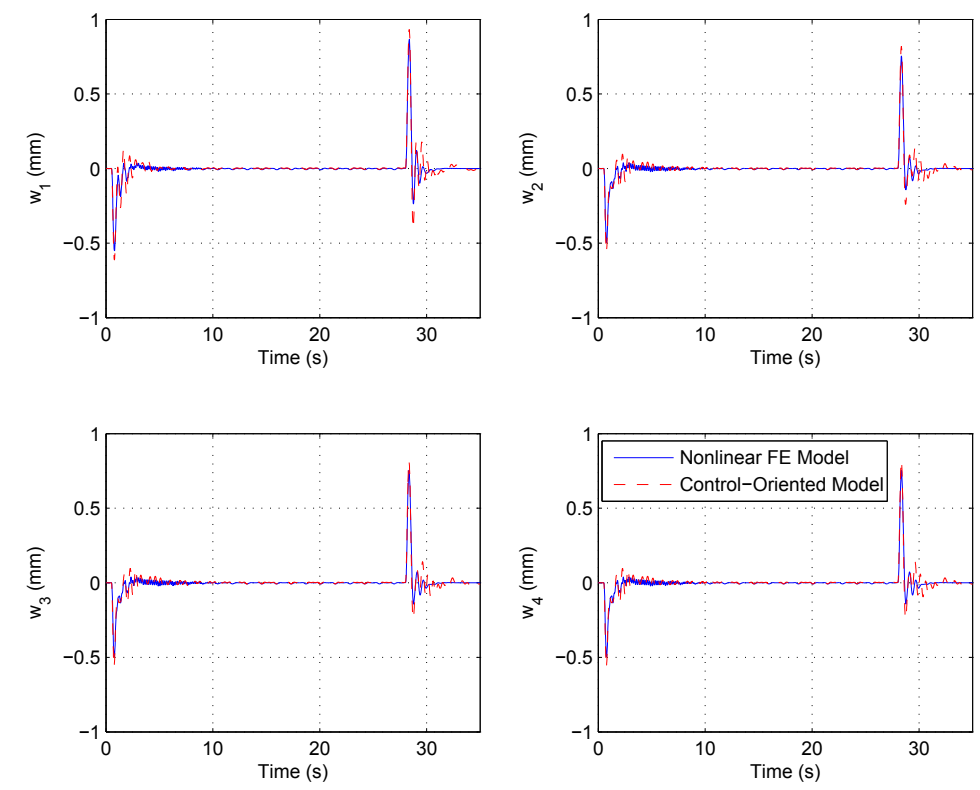

Fig. 14 Transverse deflections of the membrane in the $O_{f}-x_{f} y_{f} z_{f}$ frame (Trajectory B) 

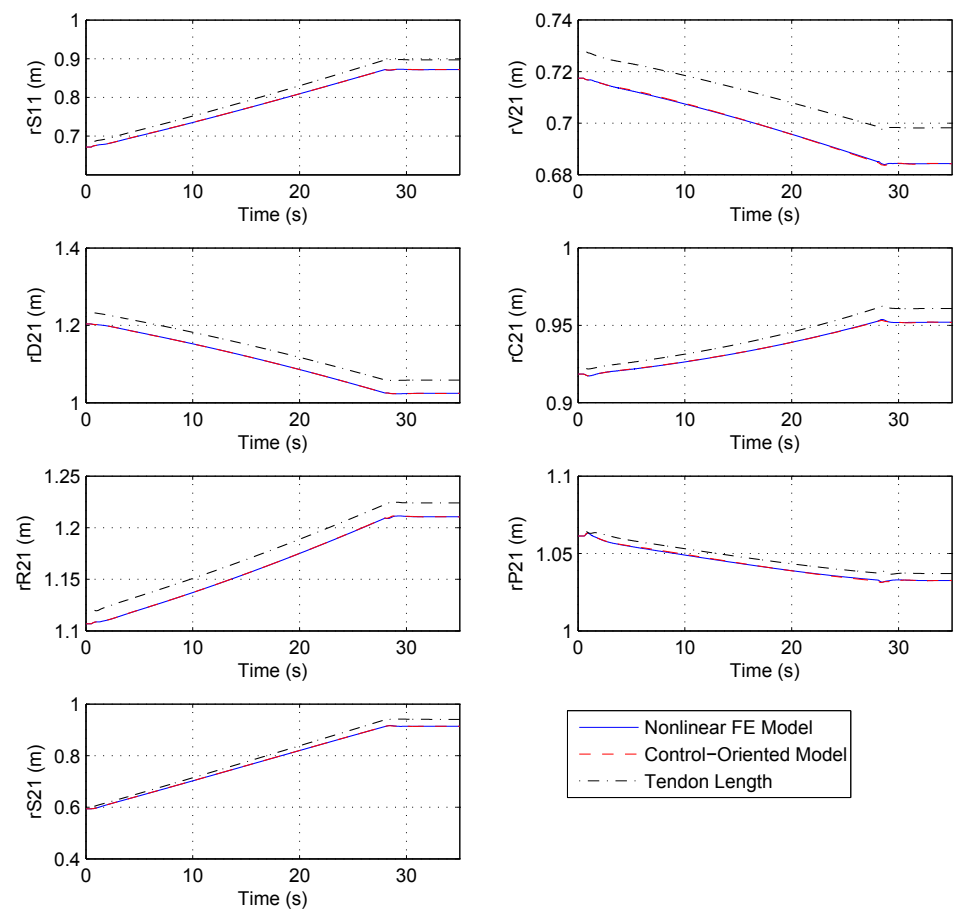

$$
\begin{array}{|l|}
\hline- \text { Nonlinear FE Model } \\
\hline--- \text { Control-Oriented Model } \\
--- \text { Tendon Length }
\end{array}
$$

\section{Fig. 15 Representative tendon rest-length variations (Trajectory B)}

Snapshots of system deployment based on the nonlinear finite element simulation results are shown in Fig. 16. It can be clearly observed that the tensegrity-membrane system experiences significant shape changes, while the membrane transverse deflections can hardly be seen. This result is in agreement with the simulation results in Fig. 14 , where the maximum membrane deflection is less than $1 \mathrm{~mm}$.

System deployment lasts for 35 seconds. According to the system responses in Figs. 12-15, it can be seen that system height changes linearly before the system reaches the final configuration, which can be also observed in Fig. 16. This result is expected, since Trajecotry B is generated by a piecewise-linear function and the LPV controller makes the system track this desired trajectory. 


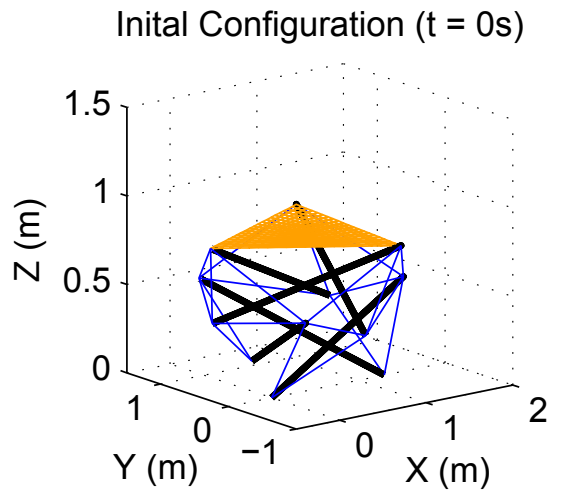

Intermediate Configuration $(t=10 \mathrm{~s})$

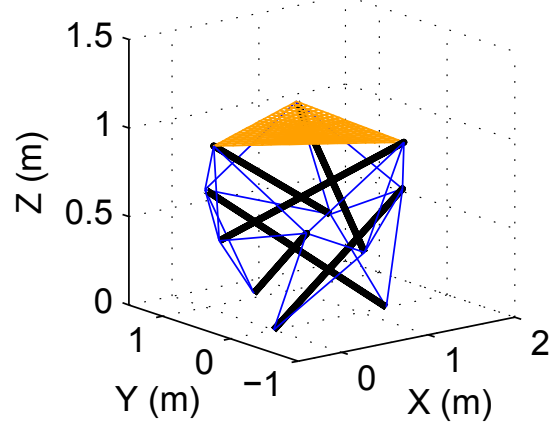

Intermediate Configuration $(t=5 s)$

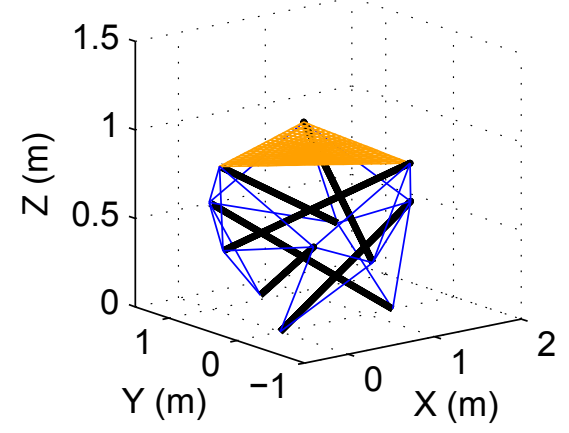

Final Configuration $(t=35 \mathrm{~s})$

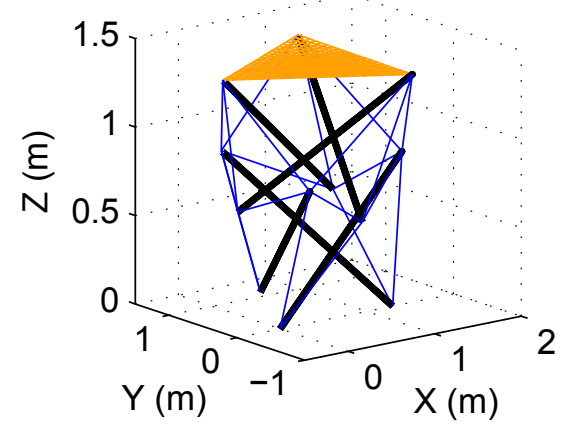

Fig. 16 Deployment sequence of a tensegrity-membrane system (Trajectory B)

The peak values of $\left|\dot{\theta}_{13}\right|$ given by the nonlinear finite element model and the control-oriented model are 0.49 deg/s and $0.50 \mathrm{deg} / \mathrm{s}$, respectively. The peak values of $\left|\dot{\psi}_{13}\right|$ given by the nonlinear finite element model and the control-oriented model are $0.24 \mathrm{deg} / \mathrm{s}$ and $0.27 \mathrm{deg} / \mathrm{s}$, respectively. Clearly, $\dot{\theta}_{13}$ and $\dot{\psi}_{13}$ are less than the bounds for the rates of $\theta_{13}$ and $\psi_{13}$ specified in Section 5, i.e. $10 \mathrm{deg} / \mathrm{s}$. This indicates that the parameter rate constraints used in LPV control design are not violated during simulations.

Yield and buckling analysis is performed for each system component and the results are presented in Table 4. It can be seen that the peak compressive load on bars is less than the critical load, the maximum von Mises stress in the membrane is less than the stress corresponding to the yield point at $3 \%$ for Kapton, and the maximum stress in tendons is less than the yield stress of Nylon. Clearly, no mechanical failure occurs during system deployment.

Table 4. Results of yield and buckling analysis (Trajectory B) 


\begin{tabular}{ccccc}
\hline & $\begin{array}{c}\text { Nonlinear finite } \\
\text { element model }\end{array}$ & $\begin{array}{c}\text { Control-oriented } \\
\text { model }\end{array}$ & Critical load & Yield stress \\
\hline $\begin{array}{c}\text { Peak compressive load } \\
\text { (bar) }\end{array}$ & $6.81 \mathrm{~N}$ & $6.85 \mathrm{~N}$ & $8357.16 \mathrm{~N}$ & $\mathrm{~N} / \mathrm{A}$ \\
$\begin{array}{c}\text { Maximum von Mises } \\
\text { stress (membrane) } \\
\begin{array}{c}\text { Maximum stress } \\
\text { (tendon) }\end{array}\end{array}$ & $1.13 \mathrm{MPa}$ & $1.48 \mathrm{MPa}$ & $\mathrm{N} / \mathrm{A}$ & $61.00 \mathrm{MPa}$ \\
\hline
\end{tabular}

\subsection{System Deployment under Sensor Noise}

In practice, feedback signals of controllers are always corrupted by sensor noise, which may degrade the control performance and eventually lead to instability for controllers without sufficient robustness. In this section, sensor noise is considered in numerical simulations to test the performance and robustness of the LPV controller. The nonlinear finite element model is used to perform simulations to test the performance of the LPV controller under the influence of sensor noise. It is assumed that, for each sensor, the signal-to-noise ratio per sample is $60 \mathrm{~dB}$, and the MATLAB function "awgn" is used to add noise to each feedback channel.

The transverse deflections of four points on the membrane are monitored to study membrane vibrations during system deployment, which are labeled $w_{1 N}, w_{2 N}, w_{3 N}$, and $w_{4 N}$. These transverse deflections are measured in the $O_{f^{-}}$ $x_{f} y_{f} z_{f}$ frame and are characterized by:

$$
\begin{aligned}
& w_{1 N}=\left.w\left(x_{f}, y_{f}\right)\right|_{x_{f}=0.86(\mathrm{~m}) ; \quad y_{f}=0.50(\mathrm{~m})} \\
& w_{2 N}=\left.w\left(x_{f}, y_{f}\right)\right|_{x_{f}=1.30(\mathrm{~m}) ; \quad y_{f}=0.75(\mathrm{~m})} \\
& w_{3 N}=\left.w\left(x_{f}, y_{f}\right)\right|_{x_{f}=0.43(\mathrm{~m}) ; \quad y_{f}=0.75(\mathrm{~m})} \\
& w_{4 N}=\left.w\left(x_{f}, y_{f}\right)\right|_{x_{f}=0.87(\mathrm{~m}) ; \quad y_{f}=0(\mathrm{~m})}
\end{aligned}
$$

\subsubsection{Trajectory $A$}

The desired trajectory parameters presented in Section 6.2.1 are also used in this case to generate the desired trajectory according to (42). The time step $\Delta t=0.01 \mathrm{~s}$, which is used in the previous section, is used in this case to numerically integrate the nonlinear finite element model as well. 
Simulation results given by the nominal system and the system with sensor noise are shown in Fig. 17 - Fig. 20. It can be observed that the LPV controller can make the system track the desired trajectory when feedback signals are corrupted by sensor noise. Compared to the results given by the nominal system, sensor noise does not have significant impact on the performance of the controller, indicating that the LPV controller has sufficient robustness. The membrane vibration time histories in Fig. 19 show that the maximum membrane deflection is less than $0.4 \mathrm{~mm}$, indicating that the membrane does not experience large amplitude vibrations. In Fig. 20, it can be seen that the tendon rest-lengths do not exceed the corresponding tendon lengths. This shows that tendons do not go slack during system deployment, which is in agreement with assumption h) in Section 3.
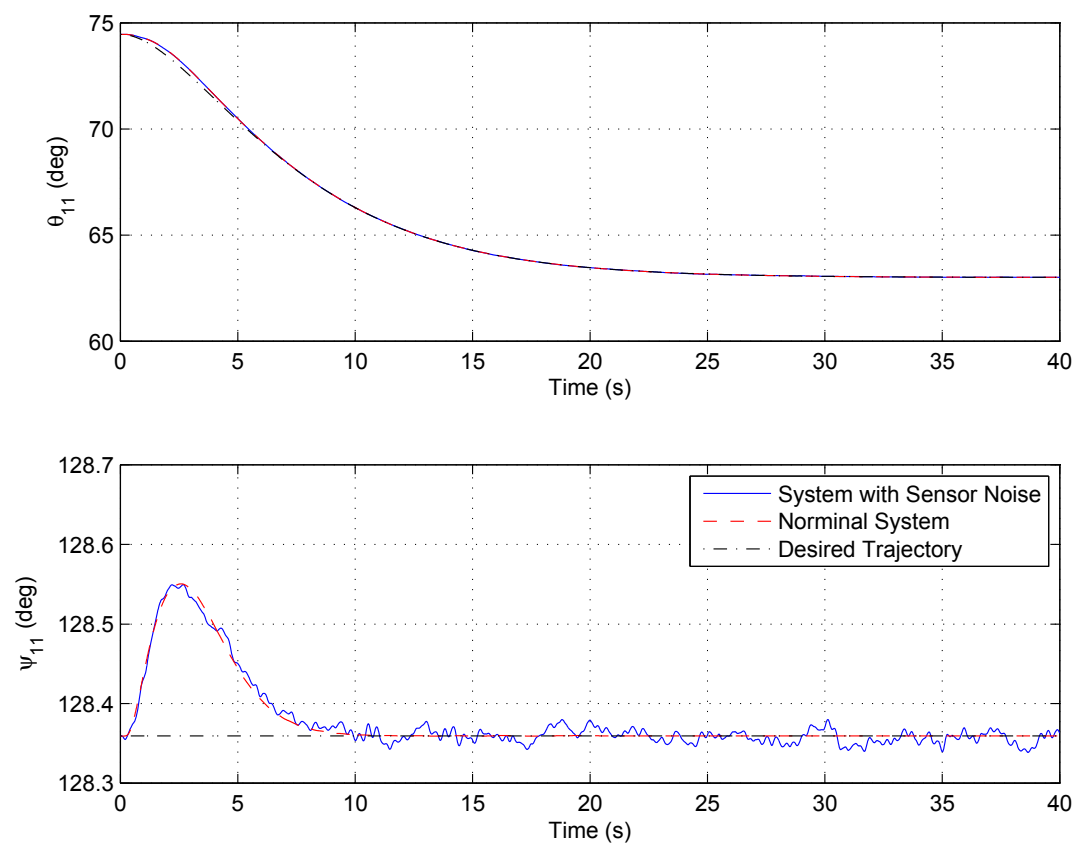

Fig. 17 Responses of bar $b_{11}$ (Trajectory A) 

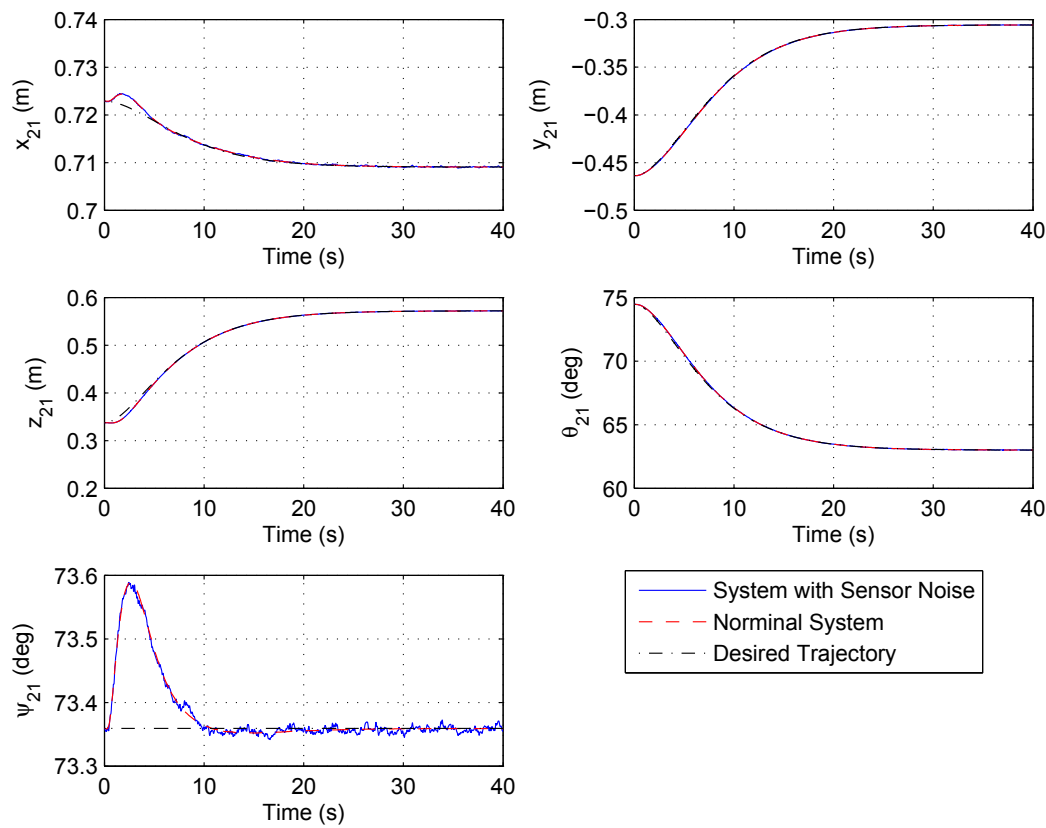

Fig. 18 Responses of bar $b_{21}$ (Trajectory A)
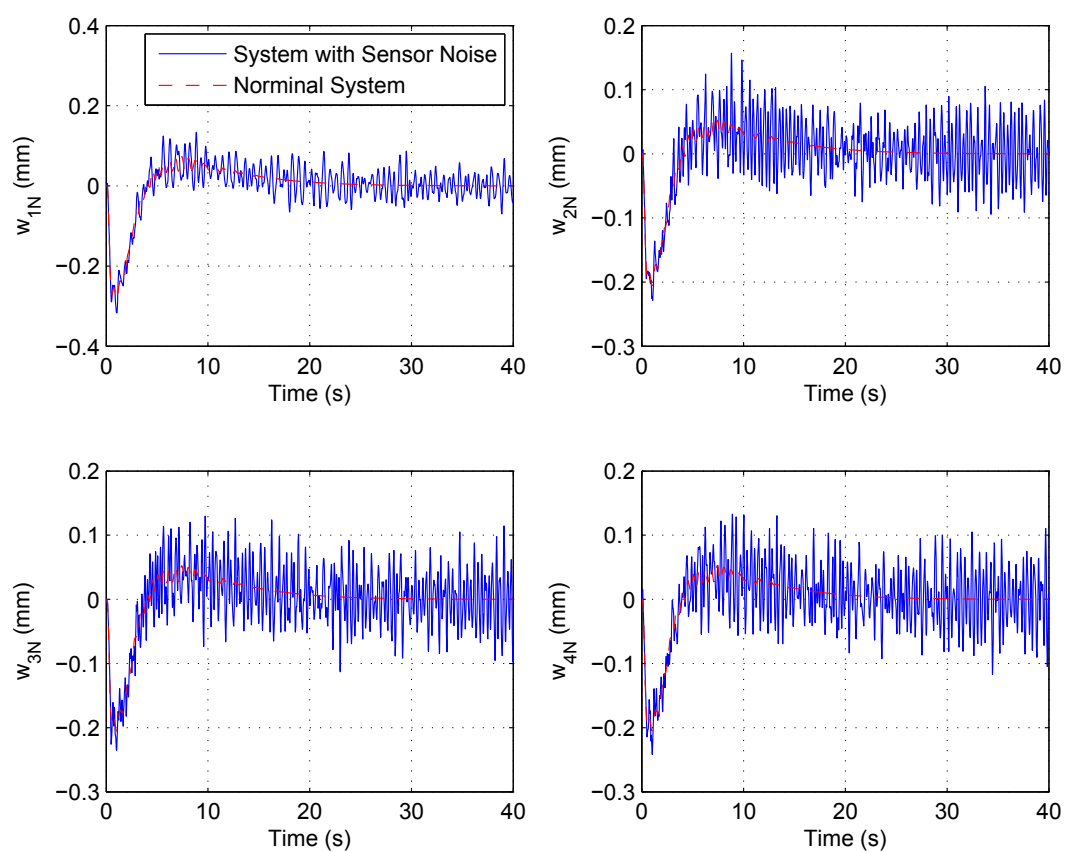

Fig. 19 Transverse deflections of the membrane in the $O_{f}-x_{f} y_{f} z_{f}$ frame (Trajectory A) 

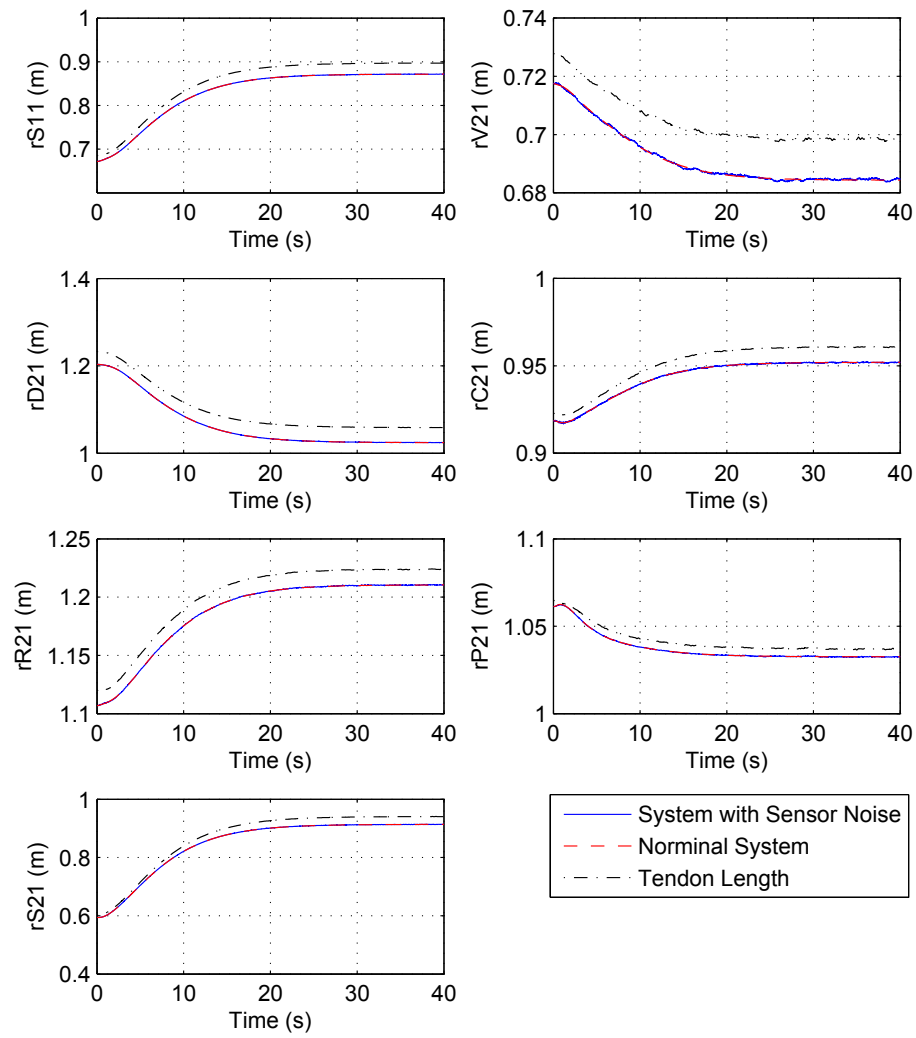

Fig. 20 Representative tendon rest-length variations (Trajectory A)

\subsubsection{Trajectory $B$}

The desired trajectory used in this section is the same as the one used in Section 6.2.2, and the time step $\Delta t=$ $0.01 \mathrm{~s}$ is used to numerically integrate the nonlinear finite element model.

Simulation results given by the nominal system and the system with sensor noise are shown in Fig. 21 - Fig. 24. Similar to the results presented in the previous section, it can be seen in Fig. 21 and Fig. 22 that the LPV controller can make the locations and attitudes of the bars track the desired trajectory when feedback signals are corrupted by sensor noise and there is no significant impact caused by sensor noise on the performance of the controller. The membrane vibration time histories shown in Fig. 23 indicate that the controller can stabilize the membrane vibrations under the influence of sensor noise. The attached membrane experiences large deflections at $t=0.5 \mathrm{~s}$ and $t=28 \mathrm{~s}$, corresponding to the discontinuities of the time derivatives of $\theta_{r}$ at these two time instants. In Fig. 24, tendon rest-lengths do not exceed the corresponding tendon lengths and the tendons do not go slack during system deployment. 

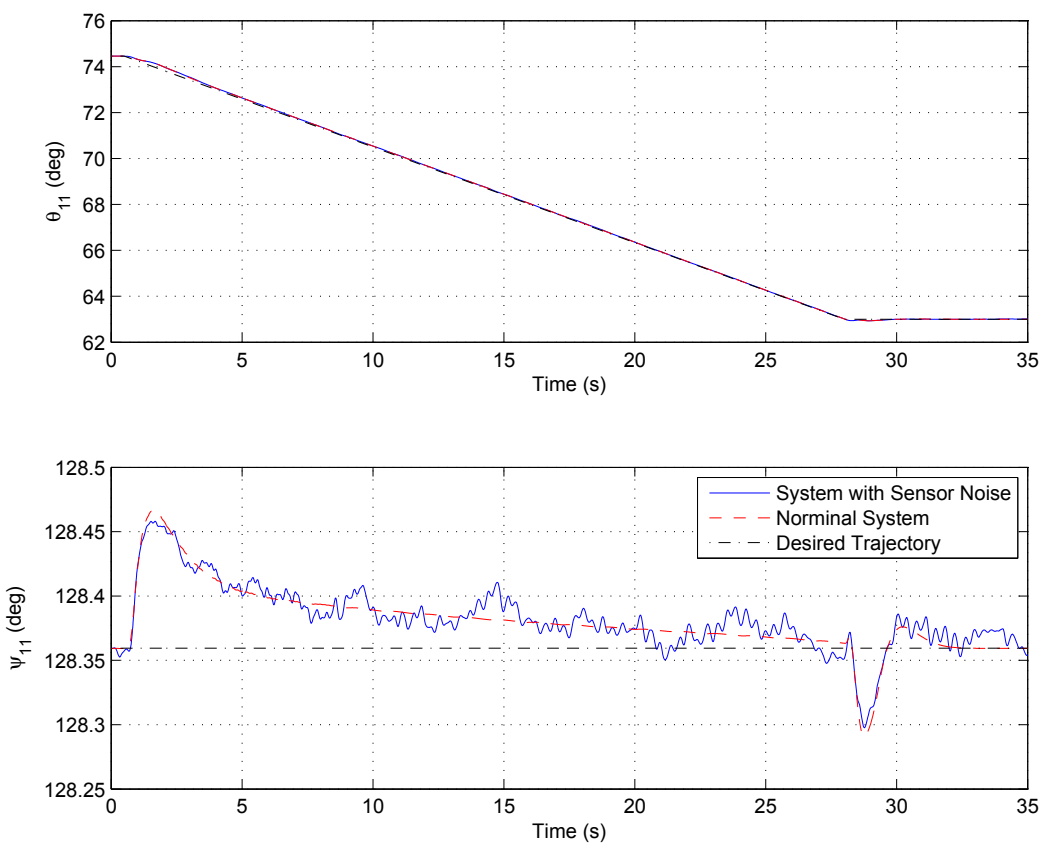

Fig. 21 Responses of bar $b_{11}$ (Trajectory B)
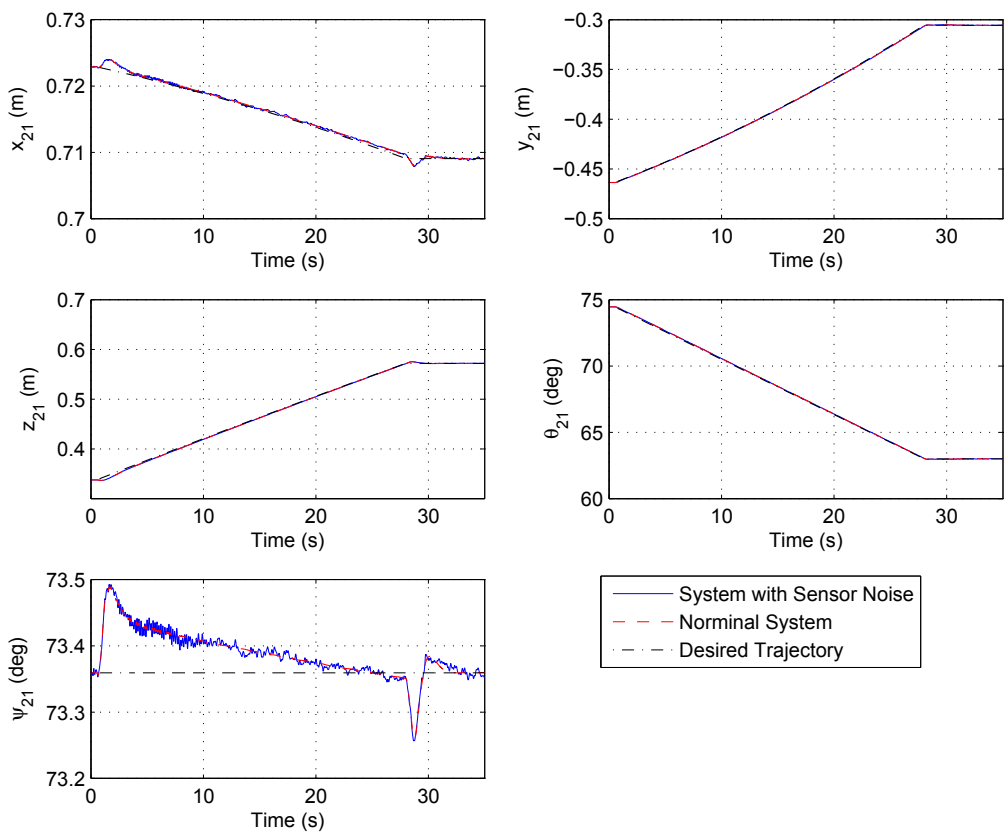

Fig. 22 Responses of bar $b_{21}$ (Trajectory B) 

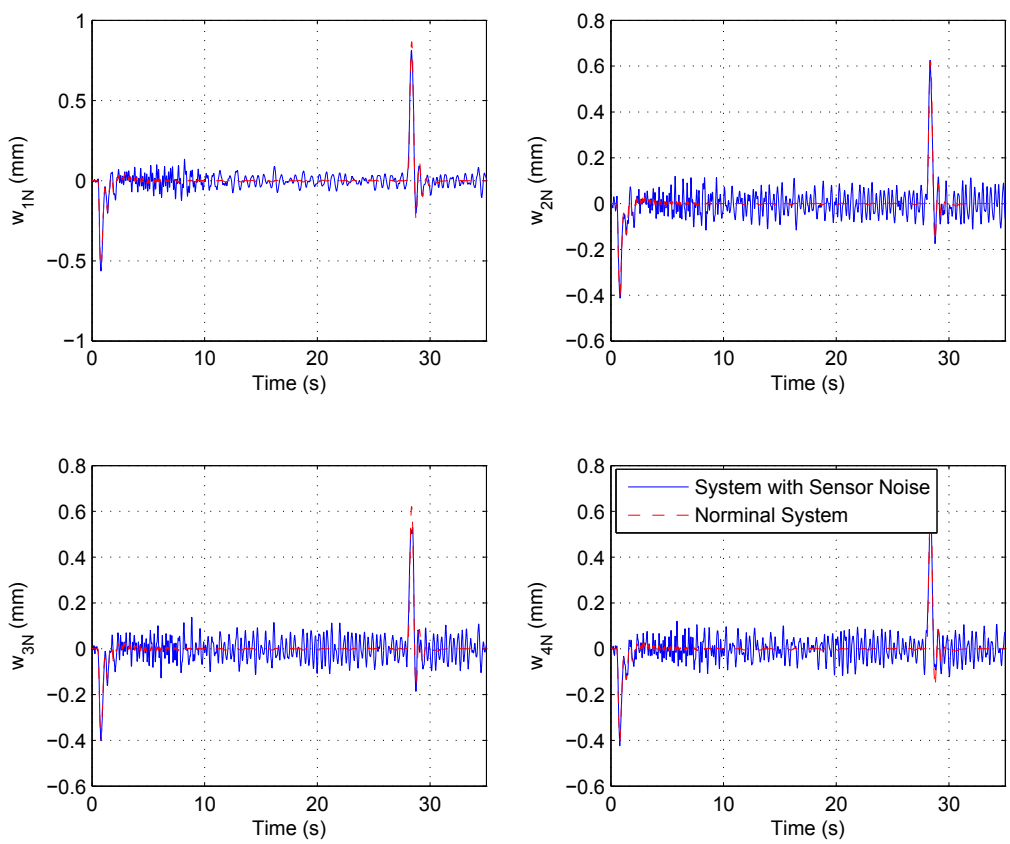

Fig. 23 Transverse deflections of the membrane in the $O_{f}-x_{f} y_{f} z_{f}$ frame (Trajectory B)
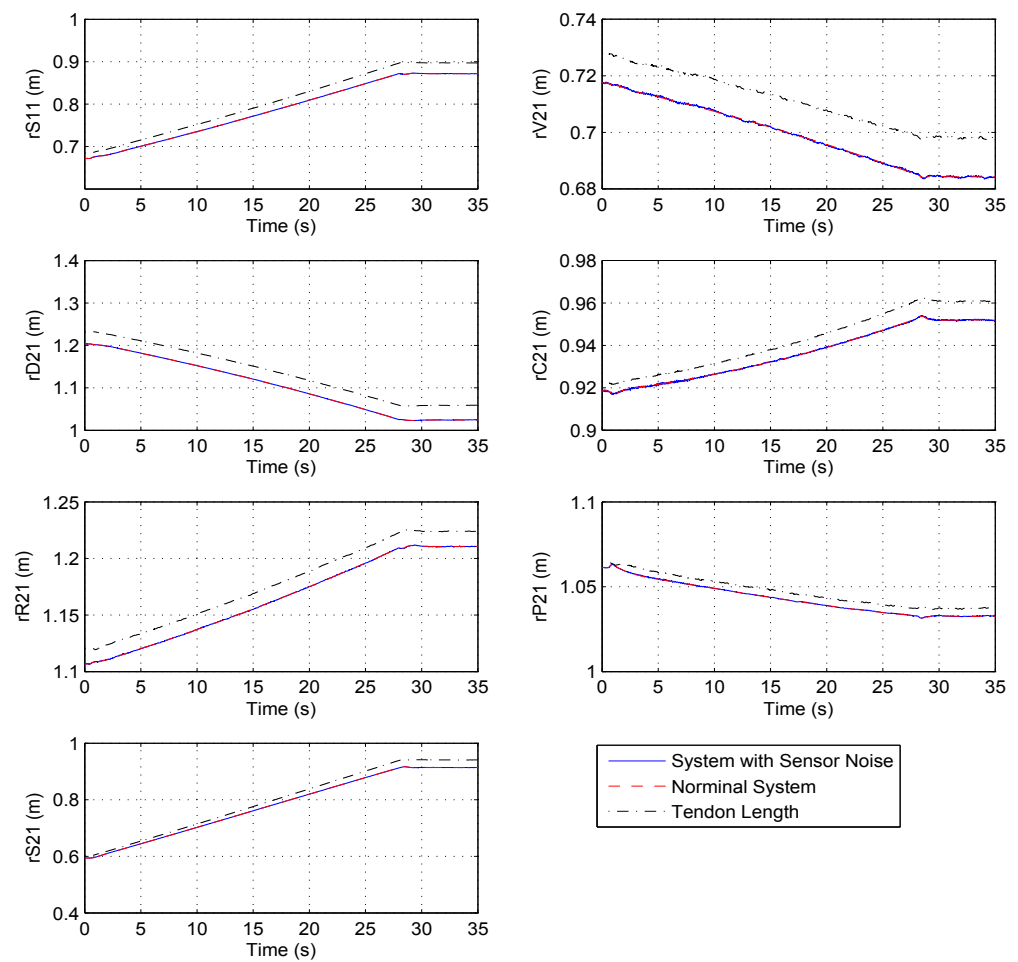

Fig. 24 Representative tendon rest-length variations (Trajectory B) 


\section{Discussions and Conclusions}

In this article, the details of designing an output-feedback LPV controller for a tensegrity-membrane system are presented. LPV synthesis is conducted based on a control-oriented model in order to achieve the desired control design objectives. To test the performance of the resulting controller, a nonlinear finite element model and the control-oriented model are used to deploy a tensegrity-membrane system from its initial configuration to its final configuration according to two desired deployment trajectories. Simulation results show that the LPV controller can make the closed-loop system track the desired trajectories. The membrane does not experience significant vibrations for Trajectory A, which is generated by a critically damped second-order system. However, Trajectory B, which is generated by a $C^{0}$ function, causes large amplitude membrane vibrations at the discontinuities of the time derivatives of this desired trajectory. Tendon rest-length variations during deployment are always less than the corresponding tendon lengths, indicating that the tendons are in tension during system deployment. This result is in agreement with a basic requirement for tensegrity-membrane system that tendons can carry only tensile loads. Moreover, simulation results given by the nonlinear finite element model and the control-oriented model are very close to each other, indicating that the control-oriented model has sufficient accuracy for control design and numerical simulations of closed-loop systems.

The LPV controller is also tested in the scenario where feedback signals are corrupted by sensor noise. The nonlinear finite element model is used in this case, and the results given by the system with sensor noise are compared with the results given by the nominal system. This analysis shows that the LPV controller can make the tensegrity-membrane system track the desired trajectories under the influence of sensor noise. The control performance is not degraded by sensor noise and all tendons are in tension. Clearly, the LPV controller designed in this work can provide good control performance and has sufficient robustness as well.

It should be pointed out that this work is intended to show the feasibility of using an LPV controller to deploy a tensegrity-membrane system. Therefore, theoretical issues related to control design and numerical simulations are studied and discussed. Topics about building a real system, conducting field tests of system deployment, and dealing with other practical issues are out of the scope of this article and will be studied in further work. 


\section{Acknowledgments}

This work was supported by the National Science Foundation (grant number: CMMI-0952558).

\section{References}

[1] G. Greschik, M. M. Mikulas, Design study of a square solar sail architecture, J. Spacecraft Rockets, 39(5) (2002) 653-661.

[2] M.M. Mikulas, A.L. Adler, Rapid structural assessment approach for square solar sails including edge support cords, in: Proceedings of the 44th AIAA/ASME/ASCE/AHS Structures, Structural Dynamics, and Materials Conference, 2003.

[3] J.C. Heald, V. Totateloff, G. Akhras, Testing of a Deployable SAR Membrane Antenna Mechanical Prototype, in: Proceedings of the 49th AIAA/ASME/ASCE/AHS/ASC Structures, Structural Dynamics, and Materials Conference, 2008.

[4] M. Leipold, H. Runge, C. Sickinger, Large SAR membrane antennas with lightweight deployable booms, in: Proceedings of the 28th ESA Antenna Workshop on Space Antenna Systems and Technologies, 2005.

[5] D.P. Cadogan, J.K. Lin, M.S. Grahne, The development of inflatable space radar reflectarrays, in: Proceedings of the 40th AIAA/ASME/ASCE/AHS/ASC Structures, Structural Dynamics, and Materials Conference and Exhibit, 1999.

[6] S. Pellegrino, Deployable membrane reflectors, in: Proceedings of the 2nd World Engineering Congress, 2002.

[7] V.S. Zolesi, P.L. Ganga, L. Scolamiero, A. Micheletti, P. Podio-Guidugli, G. Tibert, A. Donati, M. Ghiozzi, On an innovative deployment concept for large space structures, in: Proceedings of the 42nd International Conference on Environmental Systems, 2012.

[8] H. Fang, M. Lou, L. Hsia, P. Leung, Catenary systems for membrane structures, in: Proceedings of the 42nd AIAA/ASME/ASCE/AHS/ASC Structures, Structural Dynamics, and Materials Conference and Exhibit, 2001.

[9] O. Adetona, L.H. Keel, L.G. Horta, D.P. Cadogan, G.H. Sapna, S.E. Scarborough, Description of new inflatable/rigidizable hexapod structure testbed for shape and vibration control, in: Proceedings of the 43rd AIAA/ASME/ASCE/AHS/ASC Structures, Structural Dynamics and Materials Conference, 2002.

[10] O. Adetona, L.G. Horta, B.K. Taleghani, J.R. Blandino, K.J. Woods, Vibration studies of an inflatable/rigidizable hexapod structure with a tensioned membrane, in: Proceedings of the 44th AIAA/ASME/ASCE/AHS Structures, Structural Dynamics, and Materials Conference, 2003.

[11] K.T. Berger, L.G. Horta, B.K. Taleghani, Static testing of an inflatable/rigidizable hexapod structure, in: Proceedings of the 45th AIAA Structures, Structural Dynamics and Material Conference, 2004.

[12] Freeland, R., 1998. Significance of the inflatable antenna experiment technology. In: Proceedings of the 39th AIAA/ASME/ASCE/AHS/ASC Structures, Structural Dynamics, and Materials Conference.

[13] R.E. Skelton, M.C. Oliveira, Tensegrity Systems. Springer, 2009.

[14] C. Sultan, M. Corless, R.E. Skelton, Tensegrity flight simulator, J. Guid. Control Dynam. 23 (6) (2000) $1055-1064$. 
[15] C. Sultan, R.E. Skelton, Deployment of tensegrity structures, Int. J. Solids Struct. 40 (18) (2003) 4637-4657.

[16] C. Sultan, Tensegrity deployment using infinitesimal mechanisms, Int. J. Solids Struct., 51 (2014) 3653-3668.

[17] W.J. Rugh, Analytical Framework for Gain Scheduling, IEEE Contr. Syst. 11 (1) (1990) 79-84.

[18] R.A. Nichols, R.T. Reichert, W.J. Rugh, Gain scheduling for H-infinity controllers: a flight control example, IEEE Trans. Control Syst. Technol. 1 (2) (1993) 69-79.

[19] W. Tan, A.K. Packard, G.J. Balas, Quasi-LPV modeling and LPV control of a generic missile, in: Proceedings of the 2000 American Control Conference, 2000.

[20] G.J. Balas, I. Fialho, A. Pack, J. Renfrow, C. Mullaney, On the design of LPV controllers for the F-14 aircraft lateraldirectional axis during powered approach, in: Proceedings of the 1997 American Control Conference, 1997.

[21] F. Wu, A. Packard, G. J. Balas, LPV control design for pitch-axis missile autopilots, in: Proceedings of the 34th IEEE Conference on Decision and Control, 1995.

[22] S. Yang, C. Sultan, LPV state-feedback control of a tensegrity-membrane system, in: Proceedings of the 2016 American Control Conference, 2016.

[23] S. Yang, C. Sultan, Modeling of four-bar tensegrity-membrane systems, in: Proceedings of ASME 2014 International Design Engineering Technical Conferences and Computers and Information in Engineering Conference, 2014.

[24] S. Yang, C. Sultan, Active control of four-bar tensegrity-membrane systems, in: Proceedings of ASME 2014 Dynamic Systems and Control Conference, 2014.

[25] S. Yang, C. Sultan, Modeling of tensegrity-membrane systems, Int. J. Solids Struct. 82 (2016) 125-143.

[26] K.J. Bathe, Finite Element Procedures, Upper Saddle River, NJ: Prentice-Hall, 1996.

[27] W. Soedel, Vibrations of Shells and Plates, third ed., Hoboken: Taylor \& Francis, 2004.

[28] D.T. Greenwood, Principles of Dynamics, second ed., Upper Saddle River, NJ: Prentice-Hall, 1988.

[29] F. Wu, Control of Linear Parameter Varying Systems, Ph.D. dissertation, Department of Mechanical Engineering, University of California, Berkeley, CA, May 1995.

[30] P. Apkarian, R.J. Adams, Advanced gain-scheduling techniques for uncertain systems, IEEE Trans. Control Syst. Technol. 6 (1) (1998) 21-32.

[31] W. Tan, Applications of Linear Parameter-Varying Control Theory, M.S. thesis, Department of Mechanical Engineering, University of California, Berkeley, May 1997.

[32] K.C. Park, An improved stiffly stable method for direct integration of nonlinear structural dynamic equations, J. Appl. Mech., 42 (2) (1975) 464-470.

[33] C.E. Shannon, Communication in the presence of noise, Proc. IRE, 37(1) (1949) 10-21. 
[34] K. Glover, J.C. Doyle, State-space formulae for all stabilizing controllers that satisfy an $H_{\infty}$-norm bound and relations to risk sensitivity. Syst. Contr. Letters, 11 (1988) 167-172.

[35] P. Lundstrom, S. Skogestad, Z. Wang, Performance weight selection for $H$-infinity and $\mu$-control methods, T. I. Meas. Control, 13 (5) (1991) 241-252.

[36] J. Hu, C. Bohn, H. R. Wu, Systematic $H_{\infty}$ weighting function selection and its application to the real-time control of a vertical take-off aircraft, Control Eng. Pract., 8(3) (2000) 241-252.

[37] D.W. Gu, P. H. Petkov, M. M. Konstantinov, Robust Control Design with MATLAB, Springer, 2005.

[38] I.H. Shames, C.L. Dym, Energy and Finite Element Methods in Structural Mechanics, CRC Press, 1995.

[39] DuPont, Kapton ${ }^{\circledR}$ HN General-Purpose Polyimide Film, http://www.dupont.com/content/dam/assets/productsandservices/membranes-films/assets/DEC-Kapton-HN-datasheet.pdf..

[40] Cambridge University Engineering Department, Materials Data Book, http://wwwmdp.eng.cam.ac.uk/web/library/enginfo/cueddatabooks/materials.pdf

[41] C. Sultan, Tensegrity: Sixty years of art, science, and engineering, Adv. Appl. Mech., 43 (2009) 69-145.

[42] A. Marcos, G.J. Balas, Development of linear-parameter-varying models for aircraft, J. Guid. Control Dynam., 27(2) (2004) $218-228$.

[43] S. Yang, C. Sultan, Free vibration and modal analysis of a tensegrity-membrane systems, in: Proceedings of ASME 2016 International Design Engineering Technical Conferences and Computers and Information in Engineering Conference, 2016.

[44] S. Kukathasan, S. Pellegrino, Vibration of prestressed membrane structures in air, in: Proceedings of the 43rd AIAA/ASME/ASCE/AHS/ASC Structures, Structural Dynamics, and Materials Conference and Exhibit, 2002.

[45] A.L. Adler, M.M. Mikulas, J.M. Hedgepeth, Static and dynamic analysis of partially wrinkled membranes, in: Proceedings of the 41st AIAA Structures, Dynamics and Materials Conference, 2000.

[46] S. Kukathasan, S. Pellegrino, Nonlinear vibration of wrinkled membranes, in: Proceedings of the 44th AIAA/ASME/ASCE/AHS/ASC Structures, Structural Dynamics, and Materials Conference, 2003.

[47] Y.W. Wong, S. Pellegrino, Wrinkled membranes I: experiments, J. Mech. Mater. Struct., 1(1) (2006) 3-25.

[48] Y.W. Wong, S. Pellegrino, Wrinkled membranes II: analytical models, J. Mech. Mater. Struct., 1(1) (2006) $27-61$.

[49] Y.W. Wong, S. Pellegrino, Wrinkled membranes III: numerical simulations, J. Mech. Mater. Struct., 1(1) (2006) 63-95.

[50] W.D. Reynolds, T.W. Murphey, Elastic Spiral Folding for Flat Membrane Apertures, in: Proceedings of AIAA Spacecraft Structures Conference, 2014.

[51] M. Arya, S. Pellegrino, Deployment mechanics of highly compacted thin membrane structures, in: Proceedings of the AIAA Spacecraft Structures Conference, 2014. 
[52] L. Rhode-Barbarigos, C. Schulin, N.B. Ali, R. Motro, I.F. Smith, Mechanism-based approach for the deployment of a tensegrity-ring module, J. Struct. Eng., 138(4) (2012) 539-548.

[53] N. Veuve, S.D. Safaei, I.F. Smith, Deployment of a tensegrity footbridge, J. Struct. Eng., 141(11) (2015) 04015021.

[54] K. Kim, A.K. Agogino, D. Moon, L. Taneja, A. Toghyan, B. Dehghani, V. SunSpiral, A.M. Agogino, Rapid prototyping design and control of tensegrity soft robot for locomotion, in: Proceedings of the 2014 IEEE International Conference on Robotics and Biomimetics, 2014.

[55] K. Kim, A.K. Agogino, A. Toghyan, D. Moon, L. Taneja, A.M. Agogino, Robust learning of tensegrity robot control for locomotion through form-finding, in: Proceedings of the 2015 IEEE/RSJ International Conference on Intelligent Robots and Systems, 2015.

[56] D. Hustig-Schultz, V. SunSpiral, M. Teodorescu, Morphological design for controlled tensegrity quadruped locomotion, in: Proceedings of the 2016 IEEE/RSJ International Conference on Intelligent Robots and Systems, 2016.

[57] D.S. Ramrakhyani, G.A. Lesieutre, M. Frecker, S. Bharti, Aircraft structural morphing using tendon-actuated compliant cellular trusses, J. Aircraft, 42(6) (2005) 1615-1621.

[58] H. Kobayashi, R.Ozawa, Adaptive neural network control of tendon-driven mechanisms with elastic tendons, Automatica, 39(9) (2003) 1509-1519.

[59] G. Palli, G. Borghesan, C. Melchiorri, Modeling, identification, and control of tendon-based actuation systems, IEEE Trans. Robot., 28(2) (2012) 277-290.

[60] M. E. Abdallah, R. Platt, B. Hargrave, F. Permenter, Position control of tendon-driven fingers with position controlled actuators, in: Proceedings of the 2012 IEEE International Conference on Robotics and Automation, 2012.

[61] G. Martius, R. Hostettler, A. Knoll, R. Der, Compliant control for soft robots: emergent behavior of a tendon driven anthropomorphic arm, in: Proceedings of the 2016 IEEE/RSJ International Conference on Intelligent Robots and Systems, 2016.

[62] R.E. Skelton, Deployable tendon-controlled structure, U.S. Patent 5642 590, July 1, 1997.

[63] V. Bundhoo, E. Haslam, B. Birch, E.J. Park, A shape memory alloy-based tendon-driven actuation system for biomimetic artificial fingers, part I: design and evaluation, Robotica, 27(1) (2009) 131-146.

[64] G. Gilardi, E. Haslam, V. Bundhoo, E.J. Park, A shape memory alloy based tendon-driven actuation system for biomimetic artificial fingers, part II: modelling and control, Robotica, 28(5) (2010) 675-687.

[65] H. Kim, M. Han, S. Song, S. Ahn, Soft morphing hand driven by SMA tendon wire, Composites Part B: Eng., 105 (2016), 138-148.

[66] E.J. Ruggiero, Modeling and Control of SPIDER Satellite Components, Ph.D. dissertation, Department of Mechanical Engineering, Virginia Polytechnic Institute and State University, VA, July 2005. 